\title{
The spontaneous curvature of the water-hydrophobe interface
}

\author{
Felix Sedlmeier and Roland R. Netz ${ }^{\text {a) }}$ \\ Physik Department, Technische Universität München, 85748 Garching, Germany and Fachbereich Physik, \\ Freie Universität Berlin, 14195 Berlin, Germany
}

(Received 5 July 2012; accepted 13 September 2012; published online 3 October 2012)

\begin{abstract}
The temperature-dependent solvation of hydrophobic solutes in water is investigated by largescale molecular dynamics simulations. A simultaneous fit of solvation free energies for spheres and cylinders with radii up to $R=2 \mathrm{~nm}$ yields a negative Tolman length on the order of $1 \AA$ at room temperature, equivalent to a spontaneous curvature that favors water droplets over cavities. Pronounced crossover effects of the surface free energy are analyzed in terms of higherorder curvature corrections and water-discreteness effects. (C) 2012 American Institute of Physics. [http://dx.doi.org/10.1063/1.4755753]
\end{abstract}

\section{INTRODUCTION}

The hydrophobic effect is the dominant driving force for self-assembly in the aqueous environment and spans many length scales from protein folding over macromolecular aggregation and membrane formation to macroscopic phase separation. ${ }^{1}$ In fact, the solvation of hydrophobic objects displays a characteristic crossover at a length scale of $R^{*} \approx 0.5 \mathrm{~nm},{ }^{2-4}$ that can be viewed as being associated with the discreteness of water: While small hydrophobes with radii $R<R^{*}$ such as noble gas atoms can be readily accommodated within the water hydrogen bonding network, mostly reducing the water configurational freedom, large hydrophobic solutes with radii $R>R^{*}$ such as oil droplets, nano particles, or dendrimers lead to a reduction of hydrogen bonds and the formation of a liquid-vapour like interface with a positive excess entropy. Also the water density around small and large hydrophobic solutes turns out to be very different, showing a characteristic density maximum in the first solvation shell around small objects and a depletion zone around large objects. ${ }^{2-4}$

It has been a long standing question how hydrophobic solvation on the nanoscale can be described by an interface model in terms of suitably defined geometrical measures that would bridge these two very different regimes of hydrophobic solvation.

In 1949, Tolman ${ }^{5}$ proposed an asymptotic curvature correction for the surface tension of a spherical cavity, based on purely thermodynamic considerations,

$$
\gamma_{\mathrm{SPH}}=\gamma_{0}(1-2 \delta / R)
$$

where $\gamma_{0}$ is the surface tension of a planar interface, $R$ is the cavity radius, and $\delta$ is the Tolman length, which can be associated with a shift between the Gibbs dividing surface (GDS) $R_{\mathrm{GDS}}$ and the surface of tension $R_{\mathrm{S}}$ in the planar limit, $\delta=\lim _{R \rightarrow \infty} R_{\mathrm{GDS}}-R_{\mathrm{S}}{ }^{6}$ A general description of the interfacial free energy of an arbitrarily shaped interface is given by

\footnotetext{
a)Electronic mail: rnetz@physik.fu-berlin.de.
}

Helfrich's phenomenological local curvature expansion, ${ }^{7}$

$$
F_{\text {Helf }}=\int d A\left[\tilde{\gamma}+2 \kappa\left(J-c_{0}\right)^{2}+\bar{\kappa} K\right],
$$

where $J=\left(1 / R_{1}+1 / R_{2}\right) / 2, K=1 /\left(R_{1} R_{2}\right)$, and $c_{0}$ are the mean, Gaussian, and spontaneous curvatures, $R_{1,2}$ are the local principal radii of curvature and $\kappa$ and $\bar{\kappa}$ are the normal and Gaussian bending rigidities. For a sphere with radius $R$, one obtains

$$
F_{\mathrm{SPH}} / A=\gamma_{0}-\frac{4 \kappa c_{0}}{R}+(2 \kappa+\bar{\kappa}) \frac{1}{R^{2}},
$$

with $\gamma_{0} \equiv \tilde{\gamma}+2 \kappa c_{0}^{2}$ and $A=4 \pi R^{2}$. Comparison of the leading terms in the expansions in powers of the curvature $1 / R$ in Eqs. (1) and (3) shows that the Tolman length can be expressed in terms of the coefficients appearing in the Helfrich curvature expansion as

$$
\delta=2 \kappa c_{0} / \gamma_{0}
$$

With the convention that the radius of a cavity (or bubble) is taken as positive (note that all cited results in this paper are adapted to this sign convention), a negative Tolman length means that the symmetry between positive and negative radii is broken such that a droplet is preferred over a cavity. A different way of expressing this is that the surface of tension is shifted towards the liquid water side of the GDS.

The Helfrich curvature expansion in Eq. (2) can be used to describe the solvation of arbitrarily shaped hydrophobic objects in water and thus forms a powerful basis for a multitude of different advanced implicit solvation models, e.g., to predict protein folding or ligand-receptor docking. ${ }^{8}$ However, the unambiguous determination of the coefficients is for several reasons not straightforward: (i) While the magnitude of $\delta$ is generally agreed to be on the order of the molecular size, not even for simple Lennard-Jones (LJ) liquids a consensus is reached about its sign, see Refs. 9 and 10 and references therein. (ii) The aforementioned crossover between small-scale and large-scale solvation caused by the water discreteness and which is not captured by the Helfrich expression for the surface free energy, occurs at roughly the same scale as the Tolman length, i.e., $R^{*} \sim \delta$, therefore 

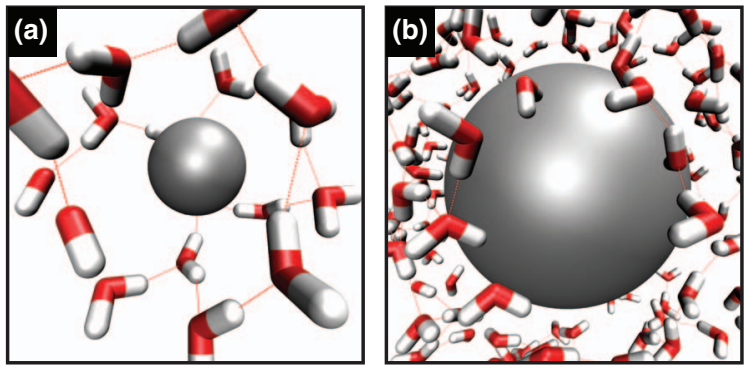

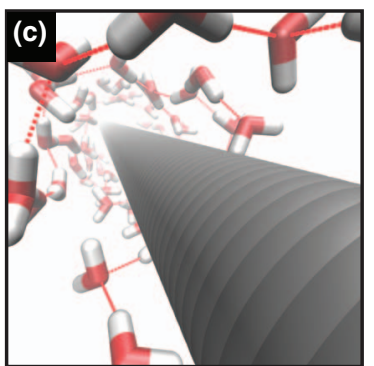

$\mathbf{R}=0.3 \mathrm{~nm}$

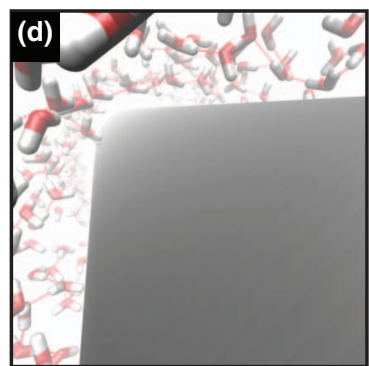

$\mathbf{R}=1.0 \mathrm{~nm}$
FIG. 1. Simulation snapshots of (a) and (b) spherical and (c) and (d) cylindrical hydrophobic solutes in water with different radii. Dashed lines indicate water hydrogen bonds.

it is not straightforward to disentangle these two lengths (the same problem hampers accurate determination of the bending rigidities $\kappa$ and $\bar{\kappa}$ as well). (iii) Furthermore, the Tolman length for water in contact with a hard solute could in principle be different from that of a liquid droplet or a bubble, where one has a liquid-vapor interface, ${ }^{11-15}$ and therefore could depend on the substrate properties in an a priori unknown manner, a point we will come back to in the following.

Using simulations and scaled particle theory (SPT), a positive Tolman length of the order of $1 \AA$ was predicted for the water-vapor interface at ambient temperature. ${ }^{16,17}$ Ashbaugh and Pratt ${ }^{4}$ find $\delta \approx 0.6 \AA$ at $T=300 \mathrm{~K}$, which decreases with increasing temperature and changes sign at $T \approx 350 \mathrm{~K}$. Huang et al. ${ }^{18}$ deduce $\delta=0.9 \AA$ at $T=298 \mathrm{~K}$ for SPC/E water from simulated solvation free energies of hydrophobic spheres. Yamamoto and Ohnishi ${ }^{19}$ on the other hand find a maximum in the surface tension of helium bubbles in water as a function of bubble radius, which, considering the curvature expansion in Eqs. (1) or (3), corresponds to a negative Tolman length, in contrast to the previous studies. Not surprisingly, the sign and magnitude of the second order curvature correction in Eq. (3), proportional to the sum of Gaussian and mean curvatures $2 \kappa+\bar{\kappa}$, is much debated as well. ${ }^{20-23}$

In the present work we employ large-scale MD simulations and determine solvation free energies of hydrophobic spherical and cylindrical solutes of radii up to $R=2 \mathrm{~nm}$ in water (see Fig. 1). For a cylinder of radius $R$, Helfrich's general curvature expansion (Eq. (2)) yields for the solvation free energy per unit area

$$
F_{\mathrm{CYL}} / A=\gamma_{0}-\frac{2 \kappa c_{0}}{R}+\frac{\kappa}{2} \frac{1}{R^{2}},
$$

and therefore using the same definition for the Tolman length Eq. (4) the equivalent of Eq. (1) is

$$
\gamma_{\mathrm{CYL}}=\gamma_{0}(1-\delta / R) \text {. }
$$

Obviously, the first-order correction term of the surface energy that depends on the Tolman length $\delta$ is different for spheres and cylinders, which provides a numerically sensitive mean to determine $\delta$ in a self-consistent manner. By considering the full radius range from zero to $R=2 \mathrm{~nm}$, we cover the crossover between the regimes where the solvation free energy scales as the solute volume, $F \sim V$ for $R<R^{*}$, and where it scales as the solute surface area, $F \sim A$ for $R>R^{*}$. Simultaneously considering spherical and cylindrical geometries turns out essential in light of the pronounced crossover effects: only the consistent and simultaneous fit of spherical and cylindrical solvation data according to Eqs. (1)-(6) allows to reliably extract the Tolman length, which is shown to be negative. Water favors droplets over cavities. By comparing the solvation free energies of spheres and cylinders, we also estimate the free energy for aggregation of spheres into cylindrical objects, which exhibits a change of sign at a radius of $R \simeq 0.3 \mathrm{~nm}$, meaning that only for large radius is the aggregation of spheres into linear cylindrical aggregates freeenergetically favored.

The solvation of small spherical hydrophobes is often used as a simplified thermodynamic model for the exposure of hydrophobic residues to water during protein unfolding. A salient feature of the solvation of small hydrophobic solutes is its temperature dependence, characterized by a negative solvation entropy at room temperature and a positive solvation heat capacity. It has been noticed that experimental solvation entropies for small spherical solutes differing in radius (e.g., noble and molecular gases, small hydrocarbons) intersect at a universal temperature around $T_{S}^{*} \simeq 400 \mathrm{~K}$, a phenomenon that goes under the name of entropy convergence. ${ }^{26}$ Based on the finding of a similar entropy convergence temperature $T_{S}^{*}$ for the unfolding of several globular proteins,${ }^{24}$ it has been argued that entropy convergence is a universal characteristic of hydrophobic solvation and from that concluded that the protein stability is dominated by the hydrophobic effect. ${ }^{25,26}$ This view was initially supported by computer simulation studies of spherical hard core solutes. ${ }^{27,28}$ Consideration of experimental data for a larger set of proteins, however, showed no evidence of entropy convergence in protein folding. ${ }^{29}$ This experimental finding can be rationalized by the spread in sizes and interaction strengths among the constituent residues as was first suggested in Ref. 4. Indeed, a recent simulation study showed that the convergence temperatures for simple spherical solutes depend sensitively on details of the solutewater interaction. ${ }^{30}$ It can be concluded that entropy convergence should only be expected for homologous series of linear molecules made of identical building blocks and is more a signature of the additivity of the solvation free energy of macromolecules than of some underlying universal solvation features.

In the present work, we go beyond previous theoretical work and investigate not only how the entropy convergence varies with the size of a solute, but also with the shape by comparing spherical and cylindrical solutes. Since a protein, when completely unfolded, might be viewed as a string of individual residues, some of which may happen to be hydrophobic depending on the sequence, a cylindrical solute might be better suited as a model for the unfolding of a protein. As 
our results show, there are salient differences between the solvation of spherical and cylindrical objects that can be rationalized as corrections due to curvature effects, as can already be seen by comparing the asymptotic expressions in Eqs. (3) and (5). One main point of our paper is to bridge the theoretical description of hydrophobic solvation for solutes with radii $R<R^{*}$, where the solvation free energy is to first order proportional to the solute volume, dominated by a loss in entropy and accompanied by an excess water adsorption, and for large solutes with radii $R>R^{*}$, where the solvation free energy is to first order proportional to the solute area, characterized by a positive excess entropy and a pronounced depletion layer, and a local curvature expansion is valid.

\section{METHODS AND MODELING}

In this section, we give a detailed account of the simulation methods and solute models that are employed in this work.

\section{A. Water model}

For all atomistic computer simulations involving water, the used water model is, of course, crucial. In the present work, we choose the extended simple point charge (SPC/E) water model,${ }^{31}$ which is widely used and has been employed in previous studies of hydrophobic solvation (see for instance Refs. 4 and 18). Due to its computational efficiency it allows simulations of large solutes necessary to obtain robust results as we will show below. We note, however, that the SPC/E model does not accurately reproduce the experimental air/water surface tension and therefore quantitative results have to be interpreted with care.

\section{B. Solute modeling}

Spherical solutes interact with water oxygen atoms via a purely repulsive Buckingham (BH) exponential potential of the form

$$
V_{\mathrm{SPH}-\mathrm{OW}}(r)=C e^{-B r},
$$

where $r$ is the distance between the center of the solute and the water oxygen atom and the parameters $C$ and $B$ are determined as follows. The decay length $1 / B$ is chosen as $1 / B$ $=0.03 \mathrm{~nm}$, corresponding to the decay length in a Buckingham exponential-6 parameterization of the water oxygen atom by Errington and Panagiotopoulos. ${ }^{32}$ The parameter $C$ is fixed by the condition that $V_{\mathrm{SPH}-\mathrm{OW}}(R)=k_{\mathrm{B}} T_{0}$, where $R$ defines the radius of the solute, $k_{\mathrm{B}}$ is the Boltzmann constant and $T_{0}=300 \mathrm{~K}$. From this definition, we obtain $C=k_{\mathrm{B}} T_{0} \exp [B R]$ and the potential can be rewritten as $V_{\mathrm{SPH}-\mathrm{OW}}(r)=k_{\mathrm{B}} T_{0} \exp [-B(r-R)]$. Using this potential form makes sure that the stiffness of the potential does not change with increasing solute size, in contrast to a repulsive Lennard-Jones potential, that becomes softer for larger solute radii. One characteristic feature of the exponential potential is its finite value for $r \rightarrow 0$, which allows very small solutes to overlap with the water molecules. We show below that this ef- fect is only significant for very small solutes, and is therefore no serious limitation for our purposes. Interactions between the solute and water hydrogen atoms are neglected.

Cylindrical solutes are modeled as a string of spheres aligned along the z-axis, with an axial separation of $\Delta z$. The cylinder has the same length as the box size in z-direction, so that by the use of periodic boundary conditions edge effects are excluded. The interaction potential of the cylindrical solute with water oxygens is therefore given by

$$
V_{\mathrm{CYL}-\mathrm{OW}}\left(r_{\|}, z\right)=C \sum_{i=1}^{N} e^{-B \sqrt{r_{\|}^{2}+\left(z-z_{i}\right)^{2}}},
$$

where $N$ is the number of spheres, $r_{\|}=\sqrt{x^{2}+y^{2}}$ is the distance from the cylinder axis, and $z_{i}=i \Delta z, i=1, \ldots, N$, are the z-positions of the spheres that form the cylinder. To obtain a rather smooth cylinder, we choose the spacing between the spheres as $\Delta z=0.025 \mathrm{~nm}$, which ensures that the corrugation of the potential along the $\mathrm{z}$-axis is negligible and we have

$$
\begin{aligned}
V_{\mathrm{CYL}-\mathrm{OW}}\left(r_{\|}\right) & \approx C \int_{-\infty}^{\infty} \frac{d z}{\Delta z} e^{-B \sqrt{r_{\|}^{2}+z^{2}}} \\
& =\frac{2 C r_{\|}}{\Delta z} K_{1}\left(B r_{\|}\right),
\end{aligned}
$$

where $K_{1}$ is the first order modified Bessel function of the second kind. Analogously to the spherical solutes, we choose $1 / B=0.03 \mathrm{~nm}$ and determine the constant $C$ by the condition $V_{\mathrm{CYL}-\mathrm{OW}}(R)=k_{\mathrm{B}} T_{0}$. From that definition, it follows that $C=\Delta z k_{\mathrm{B}} T_{0} f_{\mathrm{CYL}}(B, R)$, with $1 / f_{\mathrm{CYL}}(B, R)=2 R K_{1}(B R)$.

For comparison with our results for an exponential potential, we also calculate solvation free energies for solutes interacting via the repulsive part of a Lennard-Jones potential,

$$
V_{\mathrm{SPH}-\mathrm{SOL}}^{\mathrm{LJ}}(r)=\frac{D}{r^{12}},
$$

for a restricted range of radii, $R<0.5 \mathrm{~nm}$. The solute radii $R$ are defined analogously to the Buckingham potential by $V_{\mathrm{SPH}-\mathrm{SOL}}^{\mathrm{LJ}}(R)=k_{\mathrm{B}} T_{0}$, yielding $D=k_{\mathrm{B}} T_{0} R^{12}$.

\section{Thermodynamic integration (TI)}

The main quantity we are interested in is the solvation free energy $F$ of the spherical and cylindrical solutes. To determine $F$, the method of thermodynamic integration is used, which relates the free energy difference $\tilde{F}$ between two states I and II of a thermodynamic system, characterized by two potential energy functions $U^{\mathrm{I}}$ and $U^{\mathrm{II}}$, to the averaged derivative $\langle\partial U(\lambda) / \partial \lambda\rangle$ of an intermediate potential energy $U(\lambda)$, that connects the two states by a virtual path, i.e., $U(\lambda=0)=U^{\mathrm{I}}$ and $U(\lambda=1)=U^{\mathrm{II}}$, where $\lambda=0 \ldots 1$ is a path variable. The free energy difference $\tilde{F}$ between the two states is then given by the integral

$$
\tilde{F}=\int_{0}^{1} d \lambda\left\langle\frac{\partial U(\lambda)}{\partial \lambda}\right\rangle_{\lambda}
$$

where the average $\langle.\rangle_{\lambda}$ has to be taken for a system interacting via $U(\lambda)$. To obtain the solvation free energy of a solute, the initial state I is chosen such that the solute does not interact with the solvent, while in the final state II, the full interaction 
with the solvent is switched on. That is, the potential energy function $U^{\mathrm{I}}$ contains no solute-solvent interactions, while $U^{\mathrm{II}}$ contains the full interaction. For simulations in an isobaricisothermal ensemble, the solvation free energy contains an additional term due to the volume change, ${ }^{33}$

$$
F=\tilde{F}+k_{\mathrm{B}} T \ln \left(\frac{V^{\mathrm{II}}}{V^{\mathrm{I}}}\right),
$$

where $V^{\mathrm{I}}$ and $V^{\mathrm{II}}$ are the system volumes in state I and state II, respectively. This term accounts for the difference in the ideal gas entropy in the initial and final state of the thermodynamic integration. For the solvation of a nonpolar solute, this volume change is mainly due to the solute's volume $\Delta V$, since the water can be considered incompressible to a good approximation. If the volume of the simulation box $V$ is much larger than the volume of the solute, the correction term is small.

For large solutes it is convenient to break the thermodynamic integration into several steps with varying system sizes and thus with varying water molecule numbers, going from an initial solute radius $R^{\mathrm{I}}$ to a final radius $R^{\mathrm{II}}$ in each step, where $R^{\mathrm{I}}=0$ for the first step and $R^{\mathrm{II}}$ equals the desired solute radius in the last step. This way, smaller simulation boxes can be used for small solutes, reducing the computational cost. A summary of the intermediate radii and the corresponding box sizes used is given in Table I.

We perform linear scaling between the states, that is the potential energy function of the intermediate states is defined by

$$
U^{R^{\mathrm{I}} \rightarrow R^{\mathrm{II}}}(\lambda)=(1-\lambda) U^{R^{\mathrm{I}}}+\lambda U^{R^{\mathrm{II}}} .
$$

Since the repulsive Buckingham potential has no divergence at small distances, linear scaling is possible even for the first step, where the solute is completely decoupled in the initial state, without getting a diverging $\left\langle\partial U^{R^{\mathrm{I}} \rightarrow R^{\mathrm{I}}}(\lambda) / \partial \lambda\right\rangle$, as for a Lennard-Jones potential. ${ }^{34}$

Since the radius of the solute changes only the prefactor of the interaction potential, the potential $U^{R^{\mathrm{I}} \rightarrow R^{\mathrm{II}}}(\lambda)$ at each intermediate $\lambda$-value corresponds to a solute of intermediate radius $R(\lambda)$. Conversely, the $\lambda$-values can be chosen in such a way, that they correspond to equidistant values of the solute radius. For the integration step from $R^{\mathrm{I}}$ to $R^{\mathrm{II}}$, the intermediate $\lambda$-value corresponding to a solute of radius $R\left(R^{\mathrm{I}}<R<R^{\mathrm{II}}\right)$ is

$$
\lambda(R)=\frac{f_{\mathrm{X}}(B, R)-f_{\mathrm{X}}\left(B, R^{\mathrm{I}}\right)}{f_{\mathrm{X}}\left(B, R^{\mathrm{II}}\right)-f_{\mathrm{X}}\left(B, R^{\mathrm{I}}\right)},
$$

for the case of a spherical $(\mathrm{X}=\mathrm{SPH})$ and cylindrical $(\mathrm{X}=\mathrm{CYL})$ solute, respectively, where $f_{\mathrm{SPH}}(B, R)=\exp (B R)$ and $f_{\mathrm{CYL}}(B, R)$ is defined above (see text after Eq. (9)). The solvation free energy for a solute of radius $R$ is then given by

$$
\begin{aligned}
F(R)= & \int_{0}^{\lambda(R)} d \lambda\left\langle\frac{\partial U^{R^{\mathrm{I}} \rightarrow R^{\mathrm{II}}}(\lambda)}{\partial \lambda}\right\rangle \\
& +k_{\mathrm{B}} T \ln \left(\frac{V(R)}{V\left(R^{\mathrm{I}}\right)}\right)+F\left(R^{\mathrm{I}}\right),
\end{aligned}
$$

with $R^{\mathrm{I}}<R<R^{\mathrm{II}}$. In order to ensure proper equilibration, we calculate $\left\langle\partial U^{R^{\mathrm{I}} \rightarrow R^{\mathrm{II}}}(\lambda) / \partial \lambda\right\rangle$ for a discrete set of $\lambda$-values corresponding to radius increments of $\Delta R=0.0125 \mathrm{~nm}$. The solvation free energy is subsequently obtained by integrating a quadratic spline interpolation of the resulting data set,

TABLE I. Simulation details for the thermodynamic integration of spherical and cylindrical solutes. Shown are

\begin{tabular}{|c|c|c|c|c|c|c|c|c|}
\hline$R^{\mathrm{I}}(\mathrm{nm})$ & $R^{\mathrm{II}}(\mathrm{nm})$ & $N_{\lambda}$ & $N_{\text {sol }}$ & \multicolumn{2}{|c|}{$L^{\mathrm{II}}(\mathrm{nm})$} & $V^{\mathrm{I}}\left(\mathrm{nm}^{3}\right)$ & $V^{\mathrm{II}}\left(\mathrm{nm}^{3}\right)$ & $r_{\mathrm{c}}^{\prime}(\mathrm{nm})$ \\
\hline \multicolumn{9}{|c|}{ Spherical solutes } \\
\hline 0.0 & 0.05 & 5 & 511 & \multicolumn{2}{|c|}{2.49} & 15.5 & 15.5 & 0.9 \\
\hline 0.05 & 0.25 & 17 & 510 & \multicolumn{2}{|c|}{2.49} & 15.5 & 15.5 & 0.9 \\
\hline 0.25 & 0.5 & 21 & 862 & \multicolumn{2}{|c|}{2.98} & 26.2 & 26.6 & 1.0 \\
\hline 0.5 & 0.75 & 21 & 1318 & \multicolumn{2}{|c|}{3.47} & 40.4 & 41.7 & 1.25 \\
\hline 0.75 & 1.0 & 21 & 2064 & \multicolumn{2}{|c|}{4.06} & 64.3 & 67.0 & 1.5 \\
\hline 1.0 & 1.25 & 21 & 2659 & \multicolumn{2}{|c|}{4.47} & 85.0 & 89.5 & 1.75 \\
\hline 1.25 & 1.5 & 21 & 3554 & \multicolumn{2}{|c|}{4.98} & 116.7 & 123.3 & 2.0 \\
\hline 1.5 & 1.75 & 21 & 4546 & \multicolumn{2}{|c|}{5.46} & 153.5 & 162.8 & 2.25 \\
\hline 1.75 & 2.0 & 21 & 5843 & \multicolumn{2}{|c|}{5.99} & 202.2 & 214.6 & 2.5 \\
\hline$R^{\mathrm{I}}(\mathrm{nm})$ & $R^{\mathrm{II}}(\mathrm{nm})$ & $N_{\lambda}$ & $N_{\text {sol }}$ & $L_{z}(\mathrm{~nm})$ & $L_{x, y}^{\mathrm{II}}(\mathrm{nm})$ & $V^{\mathrm{I}}\left(\mathrm{nm}^{3}\right)$ & $V^{\mathrm{II}}\left(\mathrm{nm}^{3}\right)$ & $r_{\mathrm{c}}^{\prime}(\mathrm{nm})$ \\
\hline \multicolumn{9}{|c|}{ Cylindrical solutes } \\
\hline 0.0 & 0.05 & 5 & 506 & 2.5 & 2.48 & 15.5 & 15.5 & 0.9 \\
\hline 0.05 & 0.25 & 17 & 580 & 3.0 & 2.45 & 17.6 & 17.9 & 0.9 \\
\hline 0.25 & 0.5 & 21 & 782 & 3.0 & 2.95 & 24.1 & 26.1 & 1.0 \\
\hline 0.5 & 0.75 & 21 & 1170 & 3.5 & 3.48 & 38.3 & 42.5 & 1.25 \\
\hline 0.75 & 1.0 & 21 & 1653 & 4.0 & 4.02 & 58.1 & 64.6 & 1.5 \\
\hline 1.0 & 1.25 & 21 & 2140 & 4.5 & 4.48 & 81.2 & 90.4 & 1.75 \\
\hline 1.25 & 1.5 & 21 & 2806 & 5.0 & 5.01 & 113.4 & 125.7 & 2.0 \\
\hline 1.5 & 1.75 & 21 & 3511 & 5.5 & 5.51 & 151.2 & 167.0 & 2.25 \\
\hline 1.75 & 2.0 & 21 & 4413 & 6.0 & 6.05 & 199.9 & 219.6 & 2.5 \\
\hline
\end{tabular}
the initial and final radii of each step $R^{\mathrm{I}}$ and $R^{\mathrm{II}}$, the number of $\lambda$-values $N_{\lambda}$, the number of water molecules $N_{\text {sol }}$, the final box length $L^{\mathrm{II}}$, the initial and final volume of the simulation box $V^{\mathrm{I}}$ and $V^{\mathrm{II}}$, and the real-space cutoff radius for the solute solvent interactions $r_{\mathrm{c}}^{\prime}$. For the cylindrical solutes, the box lengths in z-direction $L_{z}$ and the final box length in $\mathrm{x} / \mathrm{y}$-direction $L_{x, y}^{\mathrm{II}}$ are listed. 

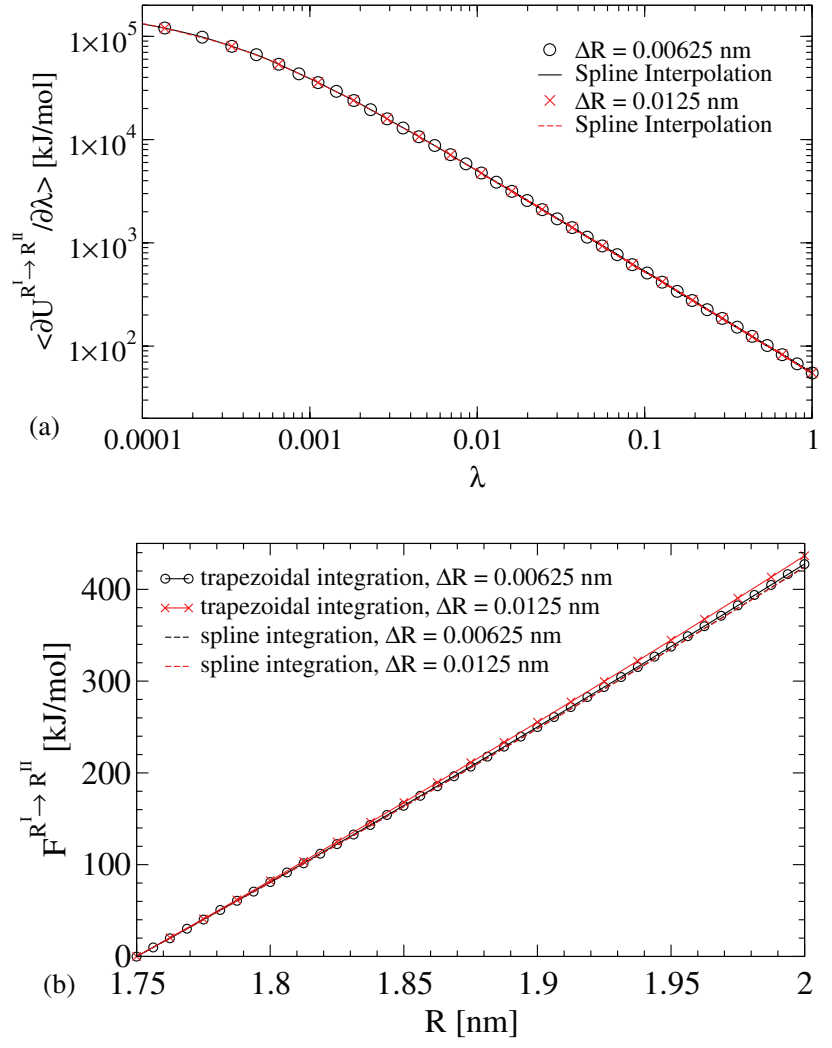

FIG. 2. (a) Average of the derivative $\left\langle\partial U^{R^{\mathrm{I}} \rightarrow R^{\mathrm{II}}} / \partial \lambda\right\rangle$ as a function of $\lambda$ for the integration step from $R^{\mathrm{I}}=1.75 \mathrm{~nm}$ to $R^{\mathrm{I}}=2.0 \mathrm{~nm}$ for a spherical solute at $T=300 \mathrm{~K}$. Red crosses and black circles indicate $\lambda$-values corresponding to equidistant radii with an increment of $\Delta R=0.0125 \mathrm{~nm}$ and $\Delta R$ $=0.00625 \mathrm{~nm}$, respectively. The black full and red dashed lines are spline interpolations to the data sets. (b) Free energy difference with respect to the initial state $R^{\mathrm{I}}=1.75 \mathrm{~nm}$ obtained by simple trapezoidal integration of $\left\langle\partial U^{R^{\mathrm{I}} \rightarrow R^{\mathrm{II}}} / \partial \lambda\right\rangle$ for an increment of $\Delta R=0.0125 \mathrm{~nm}$ (red crosses/full line) and $\Delta R=0.00625 \mathrm{~nm}$ (black circles/full line) and by integrating the spline interpolation of both data sets (red and black dashed lines).

thereby reducing the integration error significantly. We use the TI method to calculate solvation free energies of Buckingham spheres and cylinders for radii up to $R=2.0 \mathrm{~nm}$.

To check the accuracy of our integration procedure, we performed more finely resolved calculations using twice the number of $\lambda$-values, corresponding to a smaller radius increment of $\Delta R=0.00625 \mathrm{~nm}$, for the integration step $R^{\mathrm{I}}=1.75 \mathrm{~nm} \rightarrow R^{\mathrm{II}}=2.0 \mathrm{~nm}$ for the spherical solute at $T=300 \mathrm{~K}$. Figure 2(a) shows a plot of the derivative $\left\langle\partial U^{R^{\mathrm{I}} \rightarrow R^{\mathrm{II}}} / \partial \lambda\right\rangle$ as a function of $\lambda$ for the different step sizes including the interpolation. The interpolation for the coarser resolution coincides very well with the additional data points at finer resolution. In Fig. 2(b), we plot the free energy difference obtained either by simple trapezoidal integration of $\left\langle\partial U^{R^{\mathrm{I}} \rightarrow R^{\mathrm{II}}}(\lambda) / \partial \lambda\right\rangle$ (symbols and full lines) or by using the spline interpolation (dashed lines) for both data sets. It can be seen that for the trapezoidal integration there is a systematically increasing difference between the two data sets which is caused by the systematic integration error due to the concave shape of the integrand. The two curves obtained by integrating the spline interpolation lie almost perfectly on top of each other, indicating that when using the spline interpolation the coarser resolution of $\Delta R=0.0125 \mathrm{~nm}$ is sufficiently accurate.
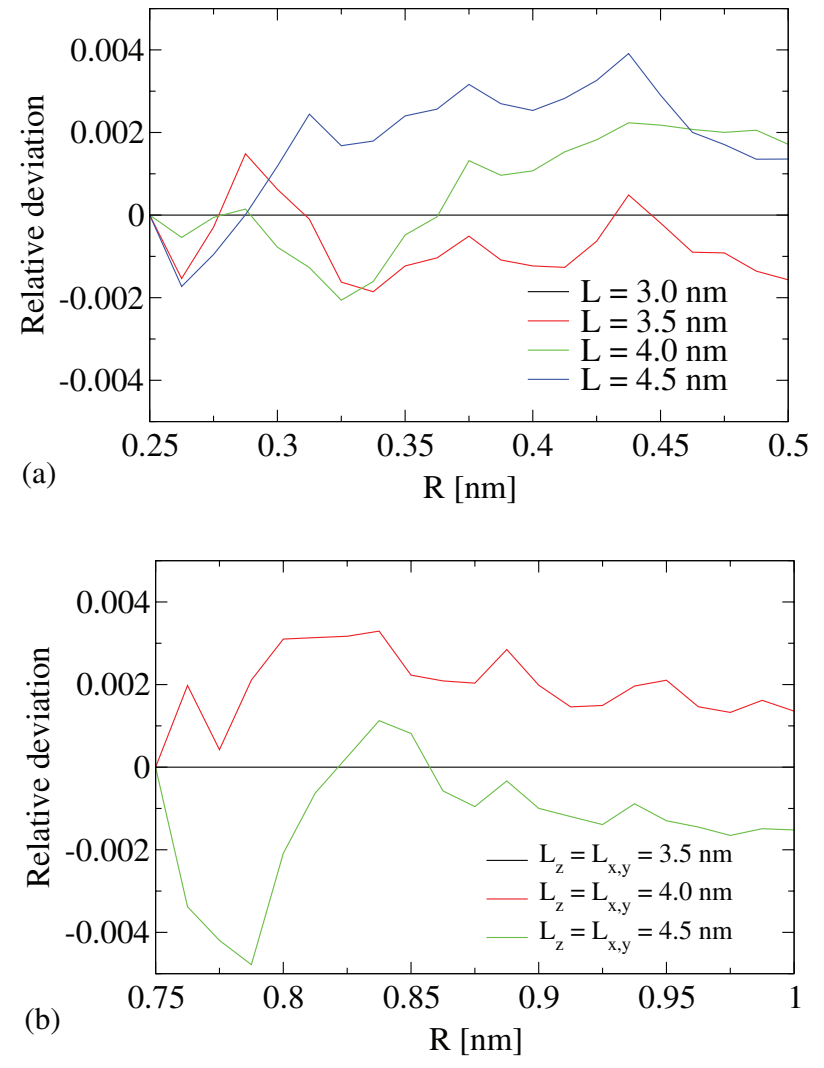

FIG. 3. Relative differences of the free energies of solvation (a) for the integration step from $R^{\mathrm{I}}=0.25 \mathrm{~nm}$ to $R^{\mathrm{II}}=0.5 \mathrm{~nm}$ for a spherical solute for different box sizes $L=3.0,3.5,4.0$, and $4.5 \mathrm{~nm}$ and (b) for the integration step from $R^{\mathrm{I}}=0.75 \mathrm{~nm}$ to $R^{\mathrm{II}}=1.0 \mathrm{~nm}$ for a cylindrical solute for box sizes of $L_{z}=L_{x, y}=3.5,4.0$, and $4.5 \mathrm{~nm}$. The differences are taken with respect to the smallest box. For the case of the cylinders, the free energy per unit length is considered. No systematic deviation is observed with increasing box size. All simulations are done at $T=300 \mathrm{~K}$ and $p=1$ bar.

\section{Finite size effects}

We further check the sensitivity of the free energy calculation to finite size effects by comparing the solvation free energies for the integration step from $R^{\mathrm{I}}=0.25 \mathrm{~nm}$ to $R^{\mathrm{II}}=0.5 \mathrm{~nm}$ for a spherical solute for different box sizes of $L$ $\approx 3.0,3.5,4.0$, and $4.5 \mathrm{~nm}$ and for the integration step from $R^{\mathrm{I}}=0.75 \mathrm{~nm}$ to $R^{\mathrm{II}}=1.0 \mathrm{~nm}$ for cylindrical solutes for box sizes of $L_{z} \approx L_{x, y} \approx 3.5,4.0$, and $4.5 \mathrm{~nm}$. Figure 3 shows the relative free energy differences with respect to the smallest box size in each case. No significant trend is seen with the system size and the relative deviations for all cases are quite small, which again evidences the high accuracy of the method. Since the simulations are done at fixed pressure, the box size $L$ (spheres) and $L_{x, y}$ (cylinders) vary slightly during each simulation. The actual box sizes at the final integration step are $L^{\mathrm{II}}=2.98,3.54,4.06$, and $4.54 \mathrm{~nm}$ for the spherical solute and $L_{x, y}^{\mathrm{II}}=3.48,4.02$, and $4.48 \mathrm{~nm}$ for the cylindrical solute, while $L_{z}=3.5,4.0$, and $4.5 \mathrm{~nm}$ is fixed.

\section{E. Test particle insertion (TPI)}

To check the results of the thermodynamic integration and to calculate solvation free energies for Lennard-Jones 
solutes for which TI is problematic, we in addition use the particle insertion method. In the case of an isobaricisothermal ensemble it is given by ${ }^{35,36}$

$$
F=-k_{\mathrm{B}} T \ln \left(\frac{\left\langle V e^{-\beta U_{\mathrm{X}-\mathrm{SOL}}}\right\rangle}{\langle V\rangle}\right),
$$

where $V$ is the volume of the system, $\beta=1 /\left(k_{\mathrm{B}} T\right)$ and the angular brackets denote an isobaric-isothermal average over configurations of the solvent system without any solute. The potential energy of the interaction between solute and solvent $U_{\mathrm{X}-\mathrm{SOL}}(\mathrm{X}=\mathrm{SPH}, \mathrm{CYL})$ is given by

$$
U_{\mathrm{X}-\mathrm{SOL}}=\sum_{i=1}^{N_{\mathrm{sol}}} V_{\mathrm{X}-\mathrm{OW}}\left(r_{i}\right)
$$

where $N_{\text {sol }}$ is the number of water molecules, $r_{\mathrm{i}}$ is the distance between the solute and the oxygen atom of the $i$ th water molecule, and $U_{\mathrm{X}-\mathrm{OW}}$ is defined in Eqs. (7) and (8), for spherical and cylindrical solutes, respectively. In contrast to the thermodynamic integration method, the particle insertion method works efficiently only for small solutes. We use the TPI method to determine the solvation free energy of Buckingham and Lennard-Jones spheres with radii $R<0.5 \mathrm{~nm}$ for comparison with the TI data (see Fig. 6).

\section{F. Planar surface tension}

The surface tension of the planar air water interface $\gamma_{\mathrm{lv}, 0}$ is determined from the anisotropy of the pressure tensor ${ }^{37}$ in simulations of a water slab surrounded by its vapour. It is given by

$$
\gamma_{\mathrm{lv}, 0}=\frac{L_{z}}{2}\left[\left\langle P_{z z}\right\rangle-\frac{\left\langle P_{x x}\right\rangle+\left\langle P_{y y}\right\rangle}{2}\right],
$$

where $L_{z}$ is the length of the simulation box in z-direction and $P_{\alpha \beta}$ denotes the components of the pressure tensor. The outer factor of $1 / 2$ accounts for the fact, that there are two interfaces present in the simulation box.

\section{G. Entropy-enthalpy decomposition}

The solvation free energy can be decomposed into entropic and enthalpic contributions by considering its temperature dependence. The solvation entropy is given by

$$
S=-\frac{\partial F}{\partial T}
$$

and the solvation enthalpy can be obtained from

$$
H=F+T S .
$$

We calculate $\Delta S$ at a given temperature $T$ by numerically taking the temperature derivative,

$$
S(T)=\frac{F(T+\Delta T)-F(T-\Delta T)}{2 \Delta T},
$$

where we use $\Delta T=20 \mathrm{~K}$. The enthalpy $H$ is then obtained from Eq. (20).

\section{H. Molecular dynamics simulations}

The necessary averages for the thermodynamic integration and particle insertion method are obtained by molecular dynamics simulations performed with a modified version of the GROMACS simulation package. ${ }^{38,39}$

The thermodynamic integration is performed in several steps. The system sizes and the particle numbers for each step are summarized in Table I. The $\lambda$-values are chosen according to Eq. (14) corresponding to equidistant solute radii with an increment of $\Delta R=0.0125 \mathrm{~nm}$. For each $\lambda$-value the system is first equilibrated for $t=50 \mathrm{ps}$ in a NVT (constant particle number, volume, and temperature) ensemble and subsequently for $t=100 \mathrm{ps}$ in a NPT (constant particle number, pressure, and temperature) ensemble in the case of the spherical solutes and a $\mathrm{NP}_{x y} \mathrm{~L}_{z} \mathrm{~T}$ (constant particle number, pressure in $\mathrm{x}$ - and $\mathrm{y}$-direction, box size in $\mathrm{z}$-direction, and temperature) ensemble in the case of the cylindrical solutes. Production runs are subsequently performed for $t=5 \mathrm{~ns}$ in the NPT (spherical solutes) or $\mathrm{NP}_{x y} \mathrm{~L}_{z} \mathrm{~T}$ (cylindrical solutes) ensemble. For the full range of solute radii $(R=0.0-2.0 \mathrm{~nm})$, this amounts to a total simulation time of $0.8 \mu$ s for each temperature. In order to account for large solutes without changing the water-water interactions, we use two different cutoff radii for the non-bonded interactions. All nonbonded real-space interactions between solvent molecules are cut off at a radius $r_{\mathrm{c}}=0.9 \mathrm{~nm}$ in most simulations, while the cutoff radius between solvent and solute particles $r_{\mathrm{c}}^{\prime}$ is chosen at least $0.5 \mathrm{~nm}$ larger than the largest solute radius (see Table I). To check the cutoff dependence of the solvation free energies, we perform additional simulations with a cutoff of $r_{\mathrm{c}}=1.8 \mathrm{~nm}$ as indicated below. Thermodynamic integrations over the full range of solute radii $(R=0.0-2.0 \mathrm{~nm})$, are performed for temperatures of $T=280,300,320$, and $360 \mathrm{~K}$ and over the range of $R=0.0-0.5 \mathrm{~nm}$ for temperatures of $T=280,300,320,340$, $360,380,400,420$, and $440 \mathrm{~K}$. For solute sizes of $R=0.05$, $0.1,0.15,0.2,0.25,0.3,0.35,0.4,0.45,0.5,0.75,1.0,1.25$, $1.5,1.75$, and $2.0 \mathrm{~nm}$ additional simulations at $T=300 \mathrm{~K}$ over $t=20 \mathrm{~ns}$ are performed to calculate solute-solvent radial distribution functions. Box sizes and solvent numbers are the same as for the thermodynamic integration (see Table I).

For test particle insertions, a system consisting of 895 water molecules is simulated at $T=300 \mathrm{~K}$ in the NPT ensemble for $t=10 \mathrm{~ns}$, after the same equilibration procedure as for each step of the thermodynamic integration.

To obtain the planar surface tension of the air/water interface, we perform simulations of a water slab in the NVT ensemble. The water slab has a thickness of $\approx 9 \mathrm{~nm}$ and contains $\approx 10000$ water molecules. It is placed in a simulation box of $6 \times 6 \times 15 \mathrm{~nm}^{3}$ and simulated for $t=10 \mathrm{~ns}$ in a NVT ensemble at temperatures of $T=280,300,320$, and $360 \mathrm{~K}$. The first 500 ps of each simulation are discarded for equilibration.

For temperature and pressure control, a Berendsen weak coupling thermostat and barostat ${ }^{40}$ with a relaxation time of $\tau=1.0 \mathrm{ps}$ is used. Long-range electrostatics are treated by 


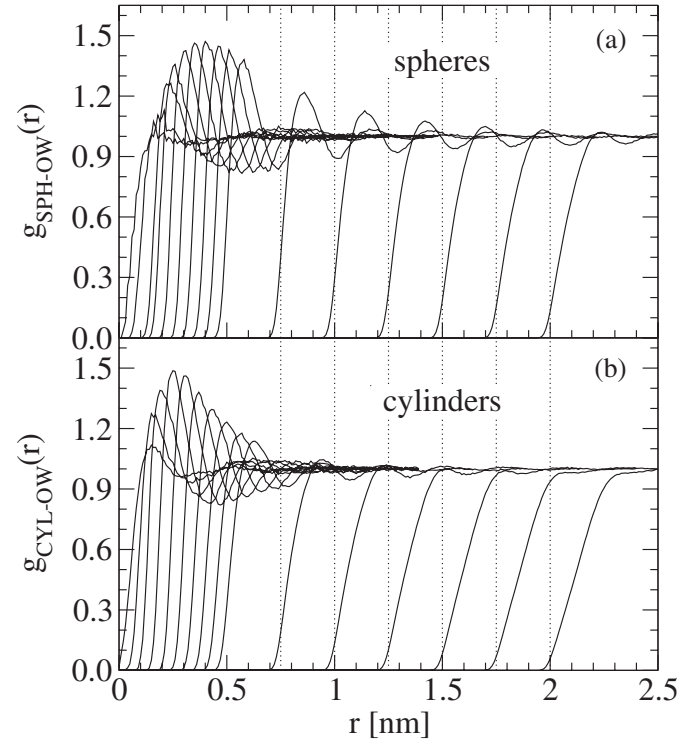

FIG. 4. Radial distribution functions (a) $g_{\mathrm{SPH}-\mathrm{OW}}(r)$ and (b) $g_{\mathrm{CYL}-\mathrm{OW}}(r)$ between solutes and water oxygen atoms for solute radii in the range $R=0.05-$ $2.0 \mathrm{~nm}$. Vertical dotted lines indicate the solute radii for $R \geq 0.75 \mathrm{~nm}$. All simulation data for SPC/E water at $T=300 \mathrm{~K}$ and $p=1$ bar.

the particle mesh Ewald summation technique ${ }^{41,42}$ with tinfoil boundary conditions.

\section{RESULTS AND DISCUSSION}

\section{A. Radius-dependent solvation}

The radial distribution functions in Fig. 4 and the solvation entropy $T S$ in Fig. 5 clearly display the gradual crossover from small-scale solvation, characterized by a negative solvation entropy $T S$ and a pronounced density peak in the first solvation shell, to large-scale solvation characterized by a monotonic density distribution around the solute, positive $T S$, and constant free energy per area $F / A$. Depending on the observable one looks at, this crossover occurs around $R^{*} \simeq 0.5-1.0 \mathrm{~nm}$ and at a significantly smaller radius for a cylinder compared to a sphere. This fact makes cylinders particularly suited for determining the Tolman length, as shown later on. Note that $F / A$ does not quite reach the independently determined planar liquid vapour surface tension $\gamma_{\mathrm{lv}, 0}$, denoted by a dotted horizontal line in Fig. 5(a), as will be discussed in detail below.

For small radii, the solvation free energy of hard-sphere solutes scales proportional to the volume. ${ }^{2,3}$ In contrast, our Buckingham spheres exhibit for very small $R$ a divergence in $F / A$ and $F / V$. This is due to the finite $r \rightarrow 0$ limit of the exponentially decaying Buckingham potential. This divergence is absent for a potential shape that becomes infinitely repulsive as $r \rightarrow 0$, as is seen in Fig. 6, where we plot the solvation free energy for spherical solutes that interact with the water oxygen atoms via a purely repulsive LJ potential (as given in Eq. (10)). Solvation free energies of the LJ-spheres are obtained by the TPI method and are shown in Fig. 6 together with solvation free energies for $\mathrm{BH}$ spheres obtained by both TI and TPI. For the LJ-spheres, F/A smoothly goes to 0 for $R$ $\rightarrow 0$, while it converges with the solvation free energy of the
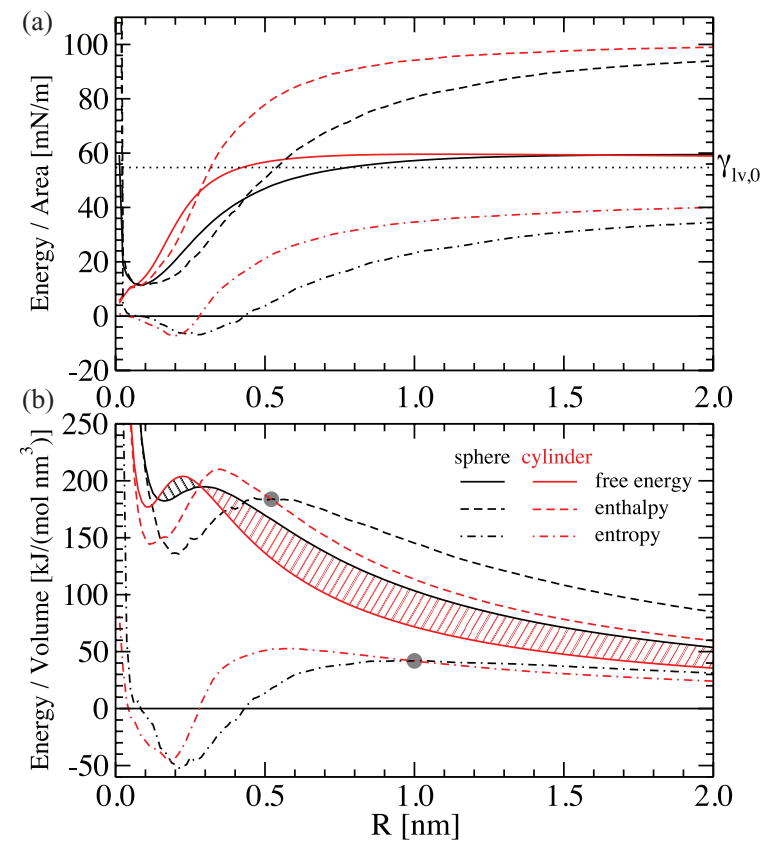

FIG. 5. Solvation free energy $F$ (full), enthalpy $H$ (dashed), and entropy $T S$ (dash-dotted lines) of spherical (black) and cylindrical (red lines) solutes as a function of the solute radius $R$, plotted (a) per unit surface area and (b) per unit volume of the solutes. The dotted horizontal line in (a) shows the independently determined surface tension of a planar liquid vapour interface $\gamma_{\mathrm{lv}, 0}$. Red and black shaded areas in (b) indicate the regions, where the difference of $F / V$ between spheres and cylinders is positive or negative. In fact, only for $R>0.3 \mathrm{~nm}$ is aggregation favored (red shaded region). The solvation enthalpic and entropic differences between spheres and cylinders change sign at $R \simeq 0.5 \mathrm{~nm}$ and $R \simeq 1.0 \mathrm{~nm}$, respectively, as indicated by filled circles. All data at $T=300 \mathrm{~K}$.

Buckingham-spheres for radii $R>0.2 \mathrm{~nm}$. The TPI data for the Buckingham-spheres agree perfectly with the Buckingham TI data for small radii. Deviations between Buckingham TPI and TI data for $R>0.4 \mathrm{~nm}$ are due to insufficient sampling, which shows that the TPI method becomes inefficient for solutes of too large size. We note that the solvation free energy $F$ of the Buckingham spherical solutes of course goes to zero for $R \rightarrow 0$, only slower than the area $A$ such that the ratio $F / A$ exhibits a weak divergence as well.

The difference between $F / V$ for spheres and cylinders in Fig. 5(b) can be viewed as the free energy change per volume upon an aggregation of spheres into a cylinder with the same radius. Although a liquid cylinder is instable with respect to an isochoric increase in radius, demonstrated by the free energy minimum at $R \rightarrow \infty$ in Fig. 5(b), this simple model for linear hydrophobic aggregation of spheres into a cylinder is still instructive: In fact, only for $R>0.3 \mathrm{~nm}$ is aggregation favored (red shaded region), for smaller $R$ the cylindrical free energy per volume is higher than the spherical one (black shaded region). This transition turns out to be driven by complex crossovers in the solvation enthalpic and entropic differences between spheres and cylinders, ${ }^{43}$ which change sign at $R \simeq 0.5 \mathrm{~nm}$ and $R \simeq 1.0 \mathrm{~nm}$, respectively, as indicated by filled circles. For $R>1.0 \mathrm{~nm}$, cylinders are entropically disfavored and enthalpically favored, while for $R$ $<0.5 \mathrm{~nm}$, cylinders are enthalpically disfavored and entropically favored. 


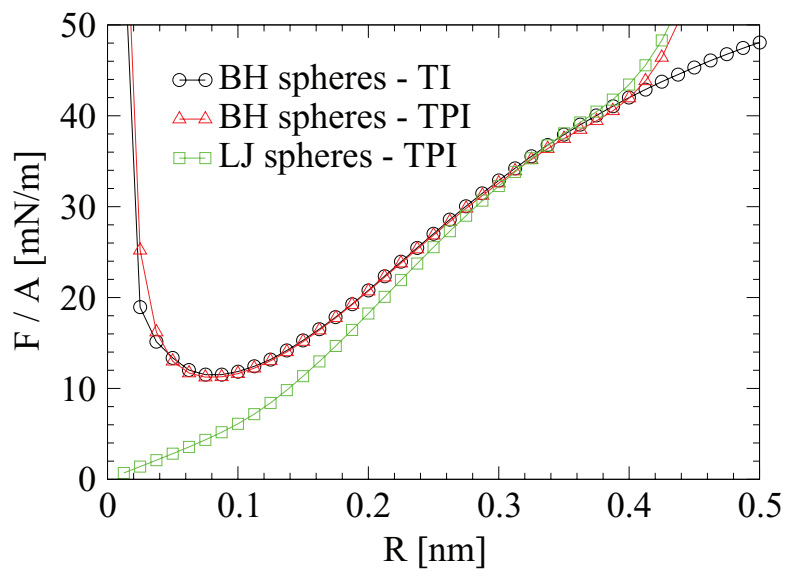

FIG. 6. Solvation free energy $F$ per solute surface area $A$ as a function of the solute radius $R$ for purely repulsive spherical solutes interacting with the water oxygen atoms either by a Buckingham (BH) (see Eq. (7)) or by a LennardJones (LJ) potential (see Eq. (10)) obtained by either the test particle insertion (TPI) or the thermodynamic integration (TI) method. All data are obtained in $\mathrm{SPC} / \mathrm{E}$ water at $T=300 \mathrm{~K}$ and $p=1 \mathrm{bar}$.

\section{B. Extracting the Tolman length}

To extract the Tolman length, in Fig. 7(a) we plot $F / A$ for $T=300 \mathrm{~K}$ as a function of the inverse radius $1 / R$ as solid lines. The data are strongly curved and fits according to the asymptotic expressions, Eqs. (1) and (6) are obviously impossible: The slopes for cylinders and spheres at $1 / R=0.5 \mathrm{~nm}^{-1}$

(a)

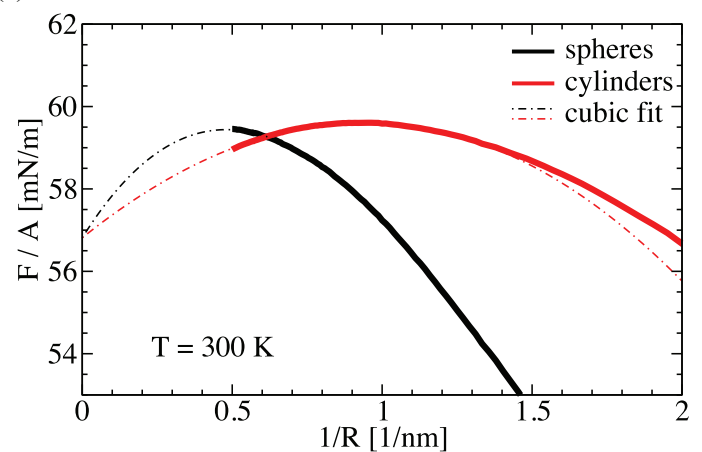

(b)

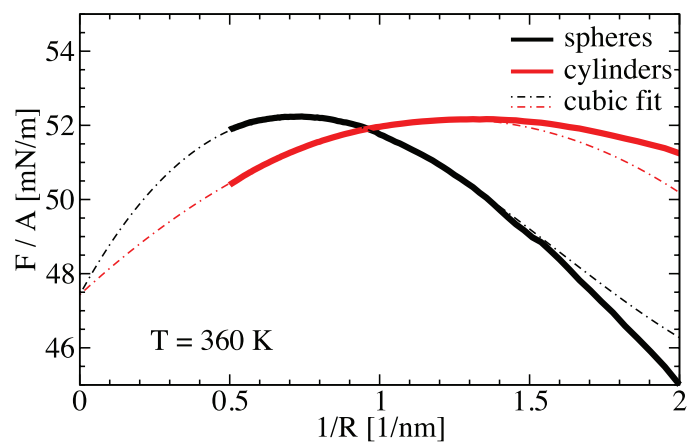

have opposite signs, so extrapolation to the limit $1 / R \rightarrow 0$ and extraction of the Tolman length (which is the negative slope in this plot) is not obvious. The situation for higher temperature $T=360 \mathrm{~K}$ in Fig. 7(b) is more favorable, since here crossovers are shifted to smaller radii ${ }^{43}$ as we will discuss in more detail in Sec. III E. Indeed, now a maximum in $F / A$ is clearly resolved in both data sets, although a linear fit according to Eqs. (1) and (6) is still not feasible, suggesting that we are still in the curvature range, where higher order corrections are important. We take the presence of higher-order corrections explicitly into account by a generalized Helfrich surface free energy that includes third-order terms in the curvature,

$$
\begin{aligned}
F_{\text {Helf }}= & \int d A\left[\tilde{\gamma}+2 \kappa\left(\frac{1}{2}\left(\frac{1}{R_{1}}+\frac{1}{R_{2}}\right)-c_{0}\right)^{2}+\bar{\kappa} \frac{1}{R_{1} R_{2}}\right. \\
& \left.+\eta\left(\frac{1}{R_{1}^{3}}+\frac{1}{R_{2}^{3}}\right)+\bar{\eta}\left(\frac{1}{R_{1} R_{2}^{2}}+\frac{1}{R_{1}^{2} R_{2}}\right)\right],
\end{aligned}
$$

where we have introduced the third-order moduli $\eta$ and $\bar{\eta}$. For a spherical solute, Eq. (22) yields

$$
F_{\mathrm{SPH}} / A=\gamma_{0}-\frac{4 \kappa c_{0}}{R}+(2 \kappa+\bar{\kappa}) \frac{1}{R^{2}}+2(\eta+\bar{\eta}) \frac{1}{R^{3}},
$$

and for a cylindrical solute,

$$
F_{\mathrm{CYL}} / A=\gamma_{0}-\frac{2 \kappa c_{0}}{R}+\frac{\kappa}{2} \frac{1}{R^{2}}+\eta \frac{1}{R^{3}} .
$$

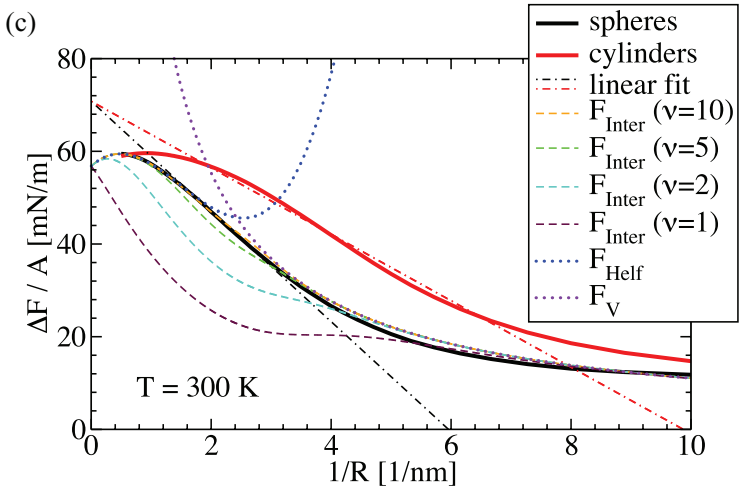

(d)

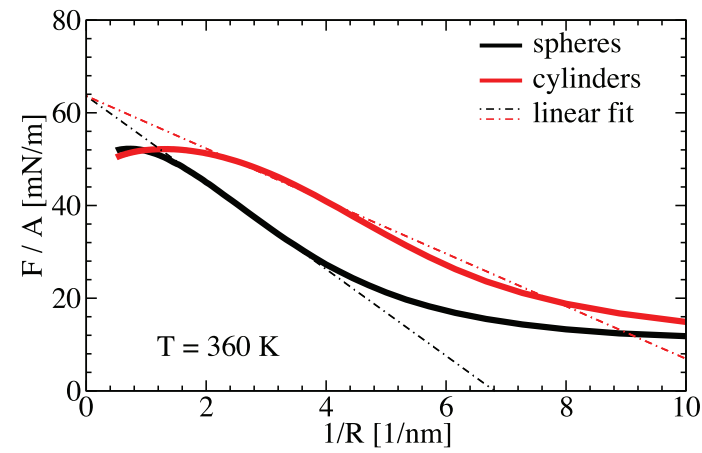

FIG. 7. Solvation free energies $F$ (full lines) per unit area of spherical (black) and cylindrical (red lines) solutes as a function of the inverse radius $1 / R$ at temperatures (a) $T=300 \mathrm{~K}$ and (b) $T=360 \mathrm{~K}$. Black and red dashed-dotted lines are cubic fits in the range $0.5 \mathrm{~nm}^{-1}<1 / R<1.4 \mathrm{~nm}^{-1}$, where Eqs. (23) and (24) are fit simultaneously to the data for spherical and cylindrical solutes. The resulting best-fit parameters are listed in Table II. (c) and (d) Same data as in (a) and (b) but for a larger range of $1 / R$. Linear fits in the region $1.5 \mathrm{~nm}^{-1}<1 / R<3 \mathrm{~nm}^{-1}$ for spheres (black dashed-dotted lines) and $3 \mathrm{~nm}^{-1}<1 / R<5 \mathrm{~nm}^{-1}$ for cylinders (red dashed-dotted lines) with the constraint of an equal surface tension are included. Additionally, in (c) the empirical interpolation function $F_{\text {Inter }}$ (Eq. (28)) for different values of the crossover exponent $v=1,2,5$, and 10 (dashed lines) together with the limiting expressions $F_{\text {Helf }}$ (Eq. (22)) (dotted blue line) and $F_{\mathrm{V}}$ (Eq. (27)) (dotted violet line) for the spherical solute is shown. 
TABLE II. Best fit parameters $\gamma_{0}, \kappa, \bar{\kappa}, c_{0}, \delta, \eta$, and $\bar{\eta}$ obtained from fitting Eqs. (23) and (24) to the spherical and cylindrical data in Figs. 7(a) and 7(b) for $(1 / R)_{\mathrm{upper}}=1.4 \mathrm{~nm}^{-1}$. The independently determined planar surface tension of the free water/vapour interface, $\gamma_{\mathrm{lv}, 0}$, is also included for comparison.

\begin{tabular}{ccccccccc}
\hline \hline$T(\mathrm{~K})$ & $\gamma_{\mathrm{lv}, 0}(\mathrm{mN} / \mathrm{m})$ & $\gamma_{0}(\mathrm{mN} / \mathrm{m})$ & $\kappa\left(10^{-21} \mathrm{~J}\right)$ & $\bar{\kappa}\left(10^{-21} \mathrm{~J}\right)$ & $c_{0}\left(\mathrm{~nm}^{-1}\right)$ & $\delta(\mathrm{nm})$ & $\eta\left(10^{-32} \mathrm{Jm}\right)$ & $\bar{\eta}\left(10^{-32} \mathrm{Jm}\right)$ \\
\hline 280 & 58.1 & 59.7 & -6.7 & -2.4 & 0.41 & -0.092 & 0.018 \\
300 & 54.7 & 56.8 & -5.8 & -2.8 & 0.50 & -0.10 & -0.23 \\
320 & 52.0 & 53.8 & -6.2 & -2.3 & 0.53 & -0.12 & -0.23 & 3.2 \\
360 & 45.5 & 47.4 & -5.4 & -2.5 & 0.69 & -0.16 & -0.35 & 3.2 \\
\hline \hline
\end{tabular}

The dashed-dotted lines in Figs. 7(a) and 7(b) show a simultaneous fit to both spherical and cylindrical data according to Eqs. (23) and (24) with the six coefficients $\gamma_{0}, \delta, \kappa, \bar{\kappa}$, $\eta$, and $\bar{\eta}$ as free parameters (fits are performed on the interval $0.5 \mathrm{~nm}^{-1}<1 / R<1.4 \mathrm{~nm}^{-1}$ ). A comparison between the third-order and second-order expressions Eqs. (23) and (24) and Eqs. (3) and (5) and the dependence on the fitting range is discussed below (see Figs. 8 and 9).

All fit parameters are summarized in Table II. The resulting Tolman length, which according to Eqs. (1) and (6) is the negative slope in the limit $1 / R \rightarrow 0$, is negative, which follows directly from the fact that the surface free energy $F / A$ goes initially up as $1 / R$ increases starting from $1 / R=0$. The value of $\delta$ is on the order of $\delta \approx-1 \AA$, implying that the spontaneous curvature is such that water favors droplets over cavities. With rising temperature, $\delta$ in fact further decreases. The maximum of $F / A$ for spheres occurs at $1 / R_{\max } \simeq 0.7 \mathrm{~nm}^{-1}$ for $T=360 \mathrm{~K}$ and extrapolated at $1 / R_{\max } \simeq 0.5 \mathrm{~nm}^{-1}$ for $T$ $=300 \mathrm{~K}$. This is consistent with previous simulations ${ }^{18}$ for spheres in the restricted radius range $1 / R>1 \mathrm{~nm}^{-1}$ that did not exhibit the maximum in $F / A$ and naturally were interpreted in terms of a positive Tolman length. Our increased radius range up to $R=2 \mathrm{~nm}$, the consideration of a whole range of temperatures, in conjunction with the cylindrical data, that show the maximum at considerably smaller radii, allows to clearly resolve the maximum in $F / A$ and thus to reliably extract the Tolman length.

The best-fit values for $\gamma_{0}$ are slightly larger than the independently determined surface tension $\gamma_{\mathrm{lv}, 0}$ of the planar liquid/vapour interface, see Table II and the data plot as a function of $R$ in Fig. 5(a). Similar behaviour for a Lennard-Jones liquid $^{9}$ was rationalized by suppressed interfacial fluctuations near a hard wall. ${ }^{44}$ Our analysis as presented in the following suggests that this deviation can alternatively be interpreted as a finite-size crossover, as follows from the dependence of the fitting results on the fitting range: Figure 8 shows fits according to Eqs. (23) and (24) including (dashed-dotted lines) and neglecting (dotted lines) the cubic term. The fitting is performed over the interval $0.5 \mathrm{~nm}^{-1}<1 / R<(1 / R)_{\text {upper }}$, where $(1 / R)_{\text {upper }}=0.6$ and $1.4 \mathrm{~nm}^{-1}$ in Figs. 8(a) and 8(b), respectively. The best fit parameters for $(1 / R)_{\text {upper }}=1.4 \mathrm{~nm}^{-1}$ from the full cubic fit correspond to the coefficients summarized in Table II.

As can be seen from Fig. 8, for small values of the curvature $1 / R$ the deviations between the quadratic and cubic fit are quite small. For larger curvature values but within the fitting range, the cubic function fits the data slightly better than the quadratic one, showing that higher order (i.e., cubic) curvature corrections do play a small but numerically significant role in the range of curvatures considered. The planar surface tension and the Tolman length obtained from the fits show a small but systematic dependence on the fitting range, as shown in Fig. 9. The trend is similar for the quadratic and cubic fit models, which shows that the behavior is rather model-independent and thus robust. In fact, the fitted surface tension $\gamma_{0}$ decreases with decreasing $(1 / R)_{\text {upper }}$ and approaches the surface tension $\gamma_{\mathrm{lv}, 0}$ of the planar liquid/vapor interface, which is denoted by a horizontal dotted line in Fig. 9(a), suggesting that the discrepancy between the $\gamma_{0}$ obtained from the fit and the surface tension obtained from simulations of a planar air/water interface $\gamma_{\mathrm{lv}, 0}$ can be explained by the finite maximal radius that is accessible in our simulations. Indeed, if we extrapolate $\gamma_{0}$ to $(1 / R)_{\text {upper }}$ $=0$, it agrees within the statistical errors with $\gamma_{\mathrm{lv}, 0}$. This means that the difference between the air-water interface and the interface between water and a hydrophobic substrate goes
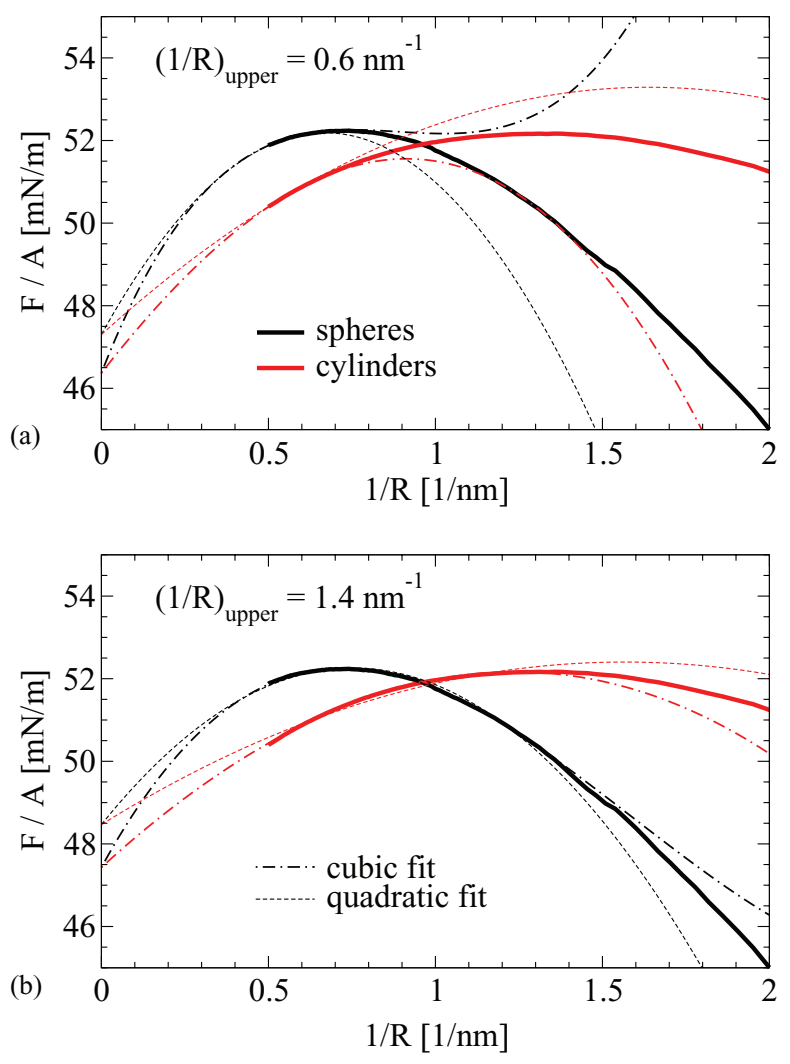

FIG. 8. Solvation free energies per area for spherical and cylindrical solutes at $T=360 \mathrm{~K}$ and $p=1 \mathrm{bar}$, simultaneously fit by Eqs. (23) and (24) either neglecting (dashed lines) or including (dashed-dotted lines) the cubic term. The fit is restricted to the range $0.5 \mathrm{~nm}^{-1}<1 / R<(1 / R)_{\text {upper }}$, where (a) $(1 / R)_{\text {upper }}$ $=0.6 \mathrm{~nm}^{-1}$ and (b) $(1 / R)_{\text {upper }}=1.4 \mathrm{~nm}^{-1}$. 


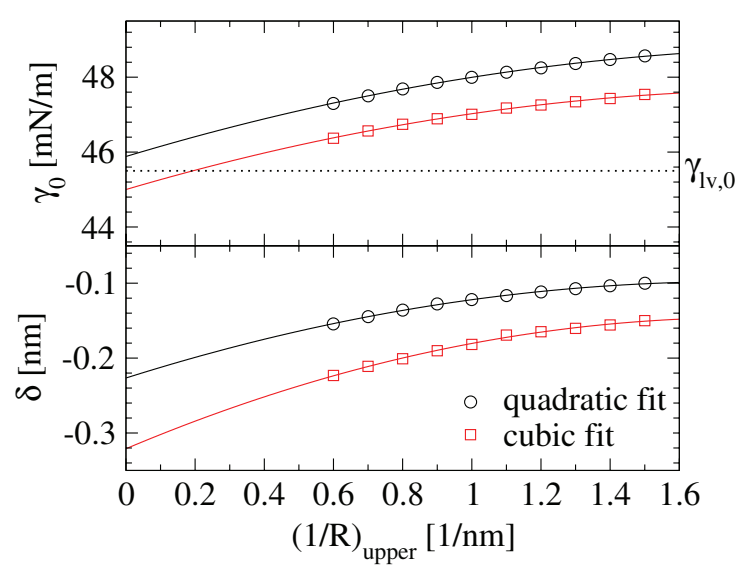

FIG. 9. Dependence of surface tension $\gamma_{0}$ and the Tolman length $\delta$ obtained from fits to the solvation free energies per area of spherical and cylindrical solutes at $T=360 \mathrm{~K}$ and $p=1$ bar for a quadratic (black circles) or cubic (red squares) fitting function (see Fig. 8) on the fitting range $0.5 \mathrm{~nm}^{-1}<1 / R$ $<(1 / R)_{\text {upper. }}$ Solid lines are quadratic fits to the data, the dotted horizontal line in (a) indicates $\gamma_{\mathrm{lv}, 0}=45.5 \mathrm{mN} / \mathrm{m}$.

to zero in the long wavelength limit, which is expected since water dewets from a hydrophobic substrate and therefore the influence of the substrate should vanish for large $R$. In that sense, the presence of a finite-size spherical or cylindrical hydrophobic solute constitutes just another finite-size effect for the interfacial free energy. We note that the analogous scaling procedure based on the data in Fig. 9(b) would predict an even more negative value for the Tolman length.

For the bending rigidities, we find negative values on the order of $\kappa, \bar{\kappa} \approx-1 k_{\mathrm{B}} T$, see Table II. This is consistent with earlier estimates based on a capillary wave spectrum analysis of MD simulations for a planar interface only when a longrange LJ correction was included. ${ }^{23}$ Note that the negativity of the bending rigidities at first sight seems like a quite robust feature of our results, indicated by the negative curvature of the data in Figs. 7(a) and 7(b), as follows by comparison with the explicit surface free energy expansion for spheres and cylinders in Eqs. (23) and (24). But one has to be careful here, since the crossover to the small scale solvation regime for $R<R^{*}$ in which $F_{\mathrm{SPH}} \sim V \sim R^{3}$ and thus $F_{\mathrm{SPH}} / A \sim R$, in conjunction with a negative Tolman length, necessitates a negative curvature of $F / A$ in a finite radius range irrespective of the sign of $\kappa$ and $\bar{\kappa}$. The consistent fit of sphere and cylinder data is of no help here, since the values of $\kappa$ and $\bar{\kappa}$ are independent. We conclude that the status of the bending rigidities is less clear than the value of the Tolman length. Since we only study solutes up to a radius of $2.0 \mathrm{~nm}$, which is on the same order as the cutoff length employed in most of our simulations, $r_{\mathrm{c}}=0.9 \mathrm{~nm}$, no strong influence of $r_{\mathrm{c}}$ would be expected. We check this explicitly by comparing results for a cylindrical solute at $T=360 \mathrm{~K}$ obtained with a cutoff length of $r_{\mathrm{c}}=0.9 \mathrm{~nm}$ and $r_{\mathrm{c}}=1.8 \mathrm{~nm}$. In Fig. 10, we show $F / A$ for both cutoff lengths rescaled by the planar air/water surface tension, which is $\gamma_{\mathrm{lv}, 0}=50.9 \mathrm{mN} / \mathrm{m}$ for $r_{\mathrm{c}}=1.8 \mathrm{~nm}$ and $\gamma_{\mathrm{lv}, 0}=45.5 \mathrm{mN} / \mathrm{m}$ for $r_{\mathrm{c}}=0.9 \mathrm{~nm}$. The slight shift, that is seen in Fig. 10, can be attributed to the statistical uncertainty of the planar surface tension, which is on the order of $2 \%$. Apart from this small deviation, the shapes of both curves are
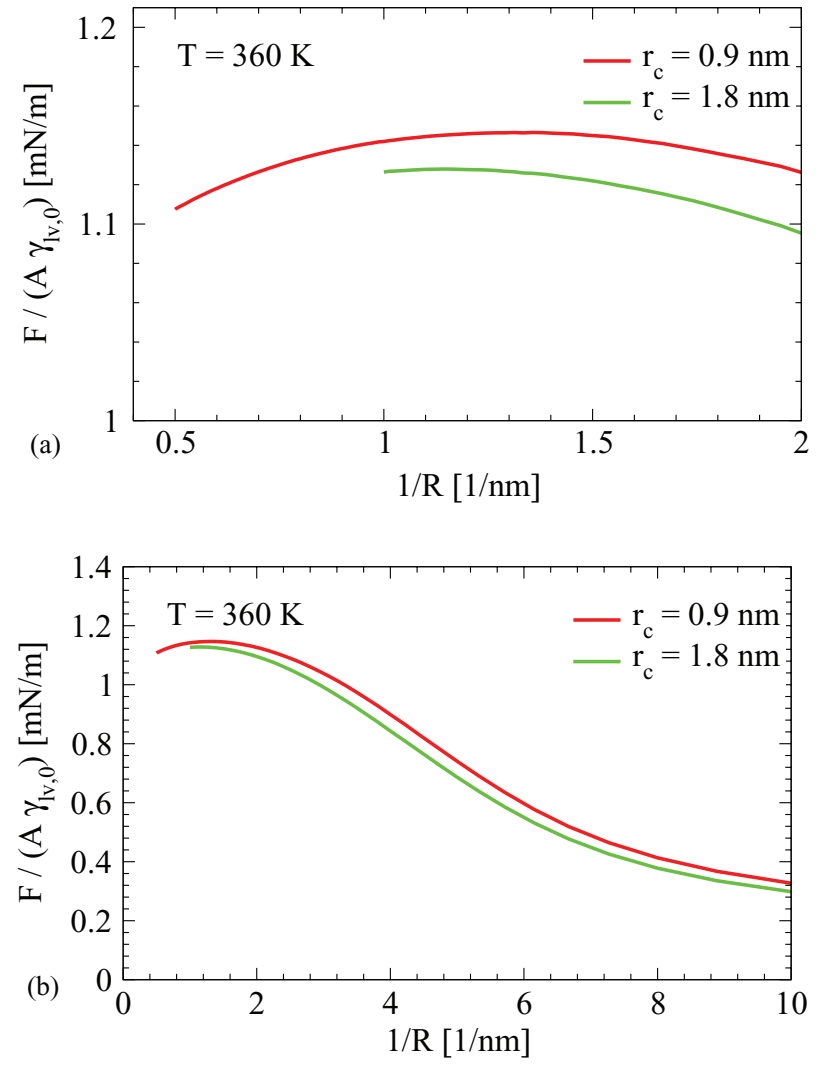

FIG. 10. Solvation free energy $F$ per area $A$ of a cylindrical solute obtained using a cutoff radius of $r_{\mathrm{c}}=0.9 \mathrm{~nm}$ (red curves) and $r_{\mathrm{c}}=1.8 \mathrm{~nm}$ (green curves) for the non-bonded water-water interactions as a function of the inverse radius $R$. The solvation free energies per area are rescaled by the planar surface tension $\gamma_{\mathrm{lv}, 0}$ of the free air/water interface obtained with the respective cutoff radii. Simulations are done at a temperature of $T=360 \mathrm{~K}$ and a pressure of $p=1 \mathrm{bar}$.

very similar, which in particular means that the Tolman length extracted from the data does not depend significantly on the Lennard-Jones cutoff length. This shows that the present way of extracting curvature and bending coefficients from solvation free energies of curved objects is an efficient alternative to the analysis of the capillary wave spectrum of a planar interface, in which case cutoff effects play a much more important role. ${ }^{23}$ Besides, the Tolman length, being proportional to the spontaneous curvature of the interface, by construction cannot be obtained from simulations of a planar interface.

\section{Dependence on radius definition}

The elastic coefficients in Eqs. (1)-(6) depend on the definition of the radius, for which there are several alternative choices besides the definition $R$ based on where the bare water-solute interaction potential equals $k_{B} T_{0}$, which we use in the main part of this paper: (i) the radius $R_{1 / 2}$ at which the water density is half of the bulk value, defined by $g_{\mathrm{X}-\mathrm{OW}}\left(R_{1 / 2}\right)$ $=1 / 2$, (ii) the radius $R_{\mathrm{GDS}}$, defined by the Gibbs dividing surface, that is, $\int d^{3} r\left[g_{\mathrm{X}-\mathrm{OW}}(r)-\theta\left(r-R_{\mathrm{GDS}}\right)\right]=0$, where $\theta(x)$ $=1$ if $x \geq 0$ and $\theta(x)=0$ if $x<0$, and (iii) the radius $R_{\mathrm{MAX}}$, defined as the first maximum of the radial distribution function $g_{\mathrm{X}-\mathrm{OW}}(r)$. In Fig. 11, we compare these different radius definitions by plotting them as a function of $R$. It is seen that, 


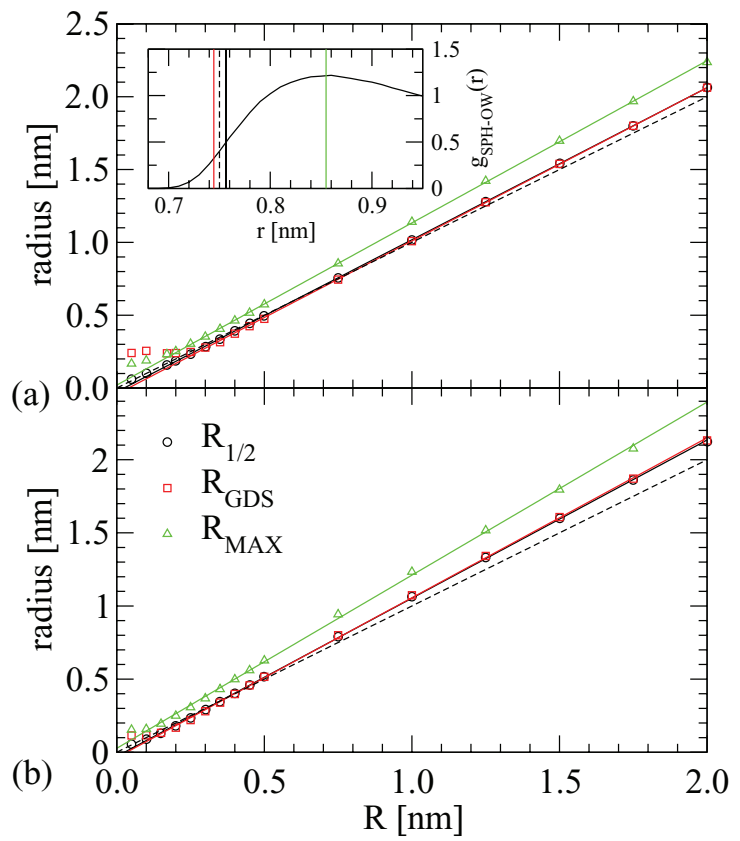

FIG. 11. Comparison of various definitions for the radius of (a) spherical and (b) cylindrical solutes in SPC/E water at $T=300 \mathrm{~K}$. Shown are the radii $R_{1 / 2}$, defined by $g_{\mathrm{X}-\mathrm{OW}}\left(R_{1 / 2}\right)=1 / 2, R_{\mathrm{GDS}}$, the radius of the Gibbs dividing surface (GDS) and $R_{\mathrm{MAX}}$, the radius of the maximum of the radial distribution function $g_{\mathrm{X}-\mathrm{OW}}(r)$, as a function of the reference radius $R$, defined by $V_{\mathrm{X}-\mathrm{OW}}(R)=k_{\mathrm{B}} T_{0}$, with $\mathrm{X}=\mathrm{SPH}$ or CYL. Solid lines are linear fits to the data. The dashed line marks the identity. In the inset, the radial distribution function $g_{\mathrm{SPH}-\mathrm{OW}}(r)$ for a spherical solute with $R=0.75 \mathrm{~nm}$ is shown and the different definitions of the radius are indicated by vertical lines.

except for $R_{\mathrm{MAX}}$, all other radii differ only slightly from $R$. For radii $R>0.2 \mathrm{~nm}$, the curves are well fit by a linear function, if we allow for a non-zero offset. For small radii $R<0.2 \mathrm{~nm}$, there are deviations from the linear dependence, which are caused by the finite $r \rightarrow 0$ limit of the Buckingham potential as discussed above. Note that the GDS based radius definition is thermodynamically the most sound definition, but requires extensive additional simulations for accurate determination. The heuristic radius definition $R$ based on the bare solute-water interaction potential we use in the main part of this paper is the most practical one, since it does not require additional simulations.

If we assume a general linear relationship between an alternative definition of the solute radius, $R^{\prime}$, and the original definition $R, R=R^{\prime}(1+\epsilon)+\Delta$, then we obtain to linear order in $1 / R^{\prime}$ for the solvation free energy of a sphere,

$$
\begin{aligned}
F_{\mathrm{SPH}} & =4 \pi R^{2} \gamma_{0}\left(1-\frac{2 \delta}{R}+\mathcal{O}\left(\frac{1}{R^{2}}\right)\right) \\
& =4 \pi R^{\prime 2} \gamma_{0}(1+\epsilon)^{2}\left(1-\frac{2(\delta-\Delta)}{R^{\prime}(1+\epsilon)}+\mathcal{O}\left(\frac{1}{R^{\prime 2}}\right)\right) .
\end{aligned}
$$

The modified Tolman length associated with the shifted radius $R^{\prime}$ is therefore given by

$$
\delta^{\prime}=\frac{\delta-\Delta}{1+\epsilon} .
$$

For $R_{\mathrm{GDS}}$ and $R_{1 / 2}$ we obtain from the fits in Fig. $11 \epsilon$ $=-0.049$ and -0.042 and $\Delta=0.040 \mathrm{~nm}$ and $0.025 \mathrm{~nm}$, respectively, with Eq. (26) leading to $\delta^{\prime}=-0.14 \mathrm{~nm}$ and $\delta^{\prime}$ $=-0.12 \mathrm{~nm}$ at $T=300 \mathrm{~K}$, we thus predict only small changes of the Tolman length.

Alternatively, using $R_{\mathrm{GDS}}$ for the radius, and employing the same cubic fitting procedure of the solvation free energy based on Eq. (22) as carried out above, we obtain the modified parameters $\gamma_{0}=52.6 \mathrm{mN} / \mathrm{m}, \kappa=-0.98 \times 10^{-21} \mathrm{~J}$, $\bar{\kappa}=-6.49 \times 10^{-21} \mathrm{~J}$, and $\delta=-0.10 \mathrm{~nm}$ at $T=300 \mathrm{~K}$. While the Tolman length does not change significantly, the bending rigidities, which constitute higher-order curvature corrections, not surprisingly do. So in line with our arguments above, our results for the bending rigidities should only be regarded as indicative.

\section{Crossover effects and empirical interpolation function}

We stress that the expressions in Eqs. (1)-(6) as well as Eqs. (23) and (24) strictly only apply to the asymptotic small-curvature regime, because the convergence of the expansion in powers of curvatures is not guaranteed. According to a conservative estimate, the applicability of the small-curvature expansions would thus be restricted to the radius range $R>R_{\max }$, with $R_{\max }$ being the radius at the maximum of the surface free energy data $F / A$ in Figs. 7(a) and 7(b), because otherwise higher-order curvature terms can take over. With $R_{\max }$ being of the order of $R_{\max } \approx 1 \mathrm{~nm}$, depending on temperature and geometry, we note that $R_{\max }$ is not much larger than $R^{*}$, which is of the order of $R^{*} \approx 0.5$ $\mathrm{nm}$, and thus the small-curvature expansion is independently invalidated by the crossover from the surface-dominated to the volume-dominated solvation scaling regime.

There are various ways of interpolating between the regime for $R<R^{*}$, where solvation scales to leading order as $F \sim V$ with corrections that can be expressed in powers of $R$, and the regime $R>R^{*}$ where solvation scales to leading order as $F \sim A$ with curvature corrections that can be written in powers of $1 / R$. Curiously, as can be seen in Figs. 7(c) and $7(\mathrm{~d})$, there is an intermediate range where $F / A$ scales approximately linear in $1 / R$ with a negative slope, the effective Tolman lengths extracted from these fits $\tilde{\delta}_{\mathrm{SPH}, \mathrm{CYL}}$ are positive and on the order of $1 \AA$, see Table III, in rough agreement with previous estimates. ${ }^{4,16-18}$ Although such a fit at intermediate radii is in principle compatible with a surface free energy functional in terms of local curvatures, it misses the turnover in the data at larger radii in a fundamental, not easily correctable way. The extracted Tolman lengths from these fits are slightly but significantly different for spheres and cylinder, which shows the inconsistency of such a fit and once more illustrates the subtleties of analyzing simulation data.

The small radius solvation behavior for $R<R^{*}$ can best be deduced from the plot of the solvation free energy per volume $F / V$ for spheres and cylinders in Fig. 5(b), where the data exhibit in a small distance range a scaling

$$
F_{\mathrm{V}} \simeq \xi V
$$


TABLE III. Best fit values for the non-asymptotic spherical and cylindrical Tolman lengths $\tilde{\delta}_{\mathrm{SPH}}$ and $\tilde{\delta}_{\mathrm{CYL}}$ from fitting Eqs. (1) and (6) to the intermediate radius range in Figs. 7(c) and 7(d) with the constraint of a common surface tension $\gamma_{0}$. The asymptotic Tolman length $\delta$ obtained from fitting Eqs. (23) and (24) to the spherical and cylindrical data is also included for comparison.

\begin{tabular}{llcc}
\hline \hline$T(\mathrm{~K})$ & $\delta(\mathrm{nm})$ & $\tilde{\delta}_{\mathrm{SPH}}(\mathrm{nm})$ & $\tilde{\delta}_{\mathrm{CYL}}(\mathrm{nm})$ \\
\hline 280 & -0.092 & 0.087 & 0.11 \\
300 & -0.10 & 0.084 & 0.10 \\
320 & -0.12 & 0.080 & 0.097 \\
360 & -0.16 & 0.073 & 0.089 \\
\hline \hline
\end{tabular}

with a prefactor $\xi \simeq 200 \mathrm{~kJ} /\left(\mathrm{mol} \mathrm{nm}^{3}\right)$ in good agreement with our estimate for SPC/E water ${ }^{45}$ and previous calculations. ${ }^{27,28}$ Note the deviations from the volume scaling for very small radii $R<0.2 \mathrm{~nm}$ which are due to our usage of the Buckingham potential, as discussed above (and which will not be treated explicitly by us in our interpolation function since it constitutes yet another scaling regime). An empirical interpolation function that encompasses both the smallscale and large-scale solvation regimes can be constructed by adding the inverse asymptotic expressions Eqs. (22) and (27) according to

$$
F_{\text {Inter }}=\left[F_{\mathrm{V}}^{-v}+F_{\text {Helf }}^{-v}\right]^{-1 / v},
$$

where the crossover exponent $v$ is positive but otherwise a freely adjustable parameter. Figure 7(c) compares this empirical interpolation function with the sphere data at $T=300 \mathrm{~K}$ for a few different values of $\nu$. As can be seen, a good overall match is obtained for a rather large value of $v$ around $v=10$, which means that the crossover is quite sharp. Such an interpolation function might be useful for simple geometries (spheres, cylinders, cylindrical networks of comparable radii, etc.) but is bound to fail for objects of more complex geometry because the crossover is enforced globally and not locally. Furthermore, in order for the interpolation formula to work, the Helfrich free energy expression (Eq. (22)) must be strictly positive over the full data range, for which cylinders require an extension to even higher order in the curvature expansion, which we do not pursue in this paper.

\section{E. Temperature dependence}

In this section, we discuss in more detail the temperature dependence of the solvation free energies of spherical and cylindrical solutes. Figure 12 shows the solvation free energy, enthalpy, and entropy for radii $R<0.5 \mathrm{~nm}$ and varying temperatures in the range $T=280-440 \mathrm{~K}$. As already mentioned, the crossover radius $R^{*}$ between the volume-like $(R$ $\left.<R^{*}\right)$ and surface-like $\left(R>R^{*}\right)$ solvation scaling regimes is smaller at higher temperatures as well as for cylinders compared to spheres. This trend is most clearly reflected in the zero-crossing of the solvation entropy in Fig. 12 (lower panels) which shifts to smaller radii with increasing temperature, noting that the small-scale solvation regime is characterized by a negative entropy associated with the entropy loss of the clathrate-like water cage around small hydrophobes. At the

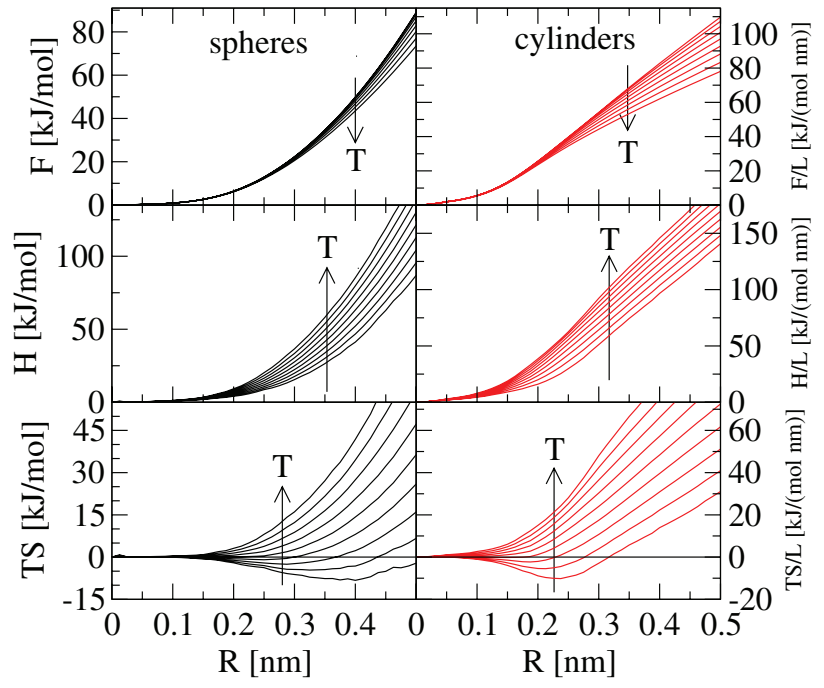

FIG. 12. Solvation free energies $F$, enthalpies $H$, and entropies $S$ for spherical and cylindrical solutes as a function of the solute radius $R$ for temperatures of $T=280,300,320,340,360,380,400,420$, and $440 \mathrm{~K}$. Arrows indicate the trends for increasing temperature. All data are obtained for SPC/E water at a constant pressure of $p=1$ bar.

same time, the depth of the minimum decreases, and above a temperature of $\approx 360 \mathrm{~K}$ vanishes completely. These findings are in line with the SPT results of Ashbaugh ${ }^{43}$ for spherical hard sphere solutes. Note that the zero-crossing of the entropy for a given temperature is associated with the crossing of two free energy curves for near-by temperatures in the upper panel. The enthalpy (middle panels) exhibits neither crossings nor sign changes.

In Fig. 13, we show in the upper panels the solvation free energy per area, $F / A$, for spherical and cylindrical solutes (circles) as a function of temperature in comparison with the planar surface tension $\gamma_{\mathrm{lv}, 0}$ (squares). Solid and dashed lines show polynomial fits to the data. For small spherical solutes in Fig. 13(a), the solvation free energies show a pronounced maximum characteristic for hydrophobic hydration. For larger solutes, the maximum of $F$ shifts to smaller temperatures. As a consequence, the entropy per area $S / A$, obtained from the temperature derivative of quadratic (for spherical solutes) or cubic (for cylindrical solutes) fits to the free energies, in the lower panels exhibits a zero-crossing at a temperature that quickly decreases with increasing particle radius. Note that $\gamma_{\mathrm{lv}, 0}$ is located in the middle of the $F / A$ curves, clearly showing that $F / A$ exhibits non-monotonic behavior both as a function of $T$ as well as a function of $R$. The fact that we see intersections of $F / A$ curves for different radii is yet another indication that the radius of maximal $F / A$ decreases with increasing temperature.

In Fig. 14, we plot the bare solvation free energies $F$ of spherical and cylindrical solutes and the corresponding entropies $S$. As the solute size increases, the maximum of $F$ shifts to smaller temperatures and the curvature becomes larger, leading to intersections of the entropies of differently sized solutes. As was noted before, ${ }^{4}$ the intersection temperature depends systematically on the size of the solutes. For cylindrical solutes in Figs. 14(c) and 14(d), the maxima of the solvation free energies per unit length (and thereby the 

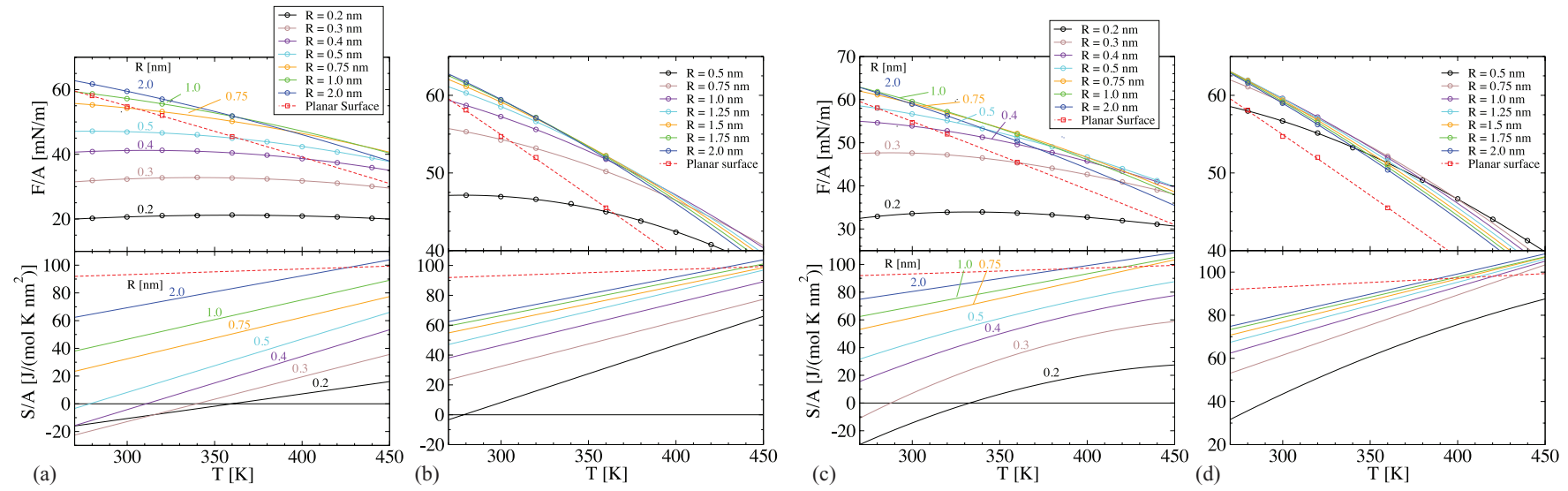

FIG. 13. Solvation free energies (top panels) and entropies (bottom panels) per area in comparison with the surface tension and entropy of a planar air/water interface as a function of temperature. (a) and (b) Solvation free energies and entropies for spherical solutes and (c) and (d) cylindrical solutes of different radii. Open circles show $F / A$ of the solutes, squares show the surface tension of the air/water interface, lines are polynomial fits to the data. Solvation entropies are obtained from fits to the free energy data by Eq. (19). All data are obtained by thermodynamic integration in SPC/E water at a constant pressure of $p=1$ bar.
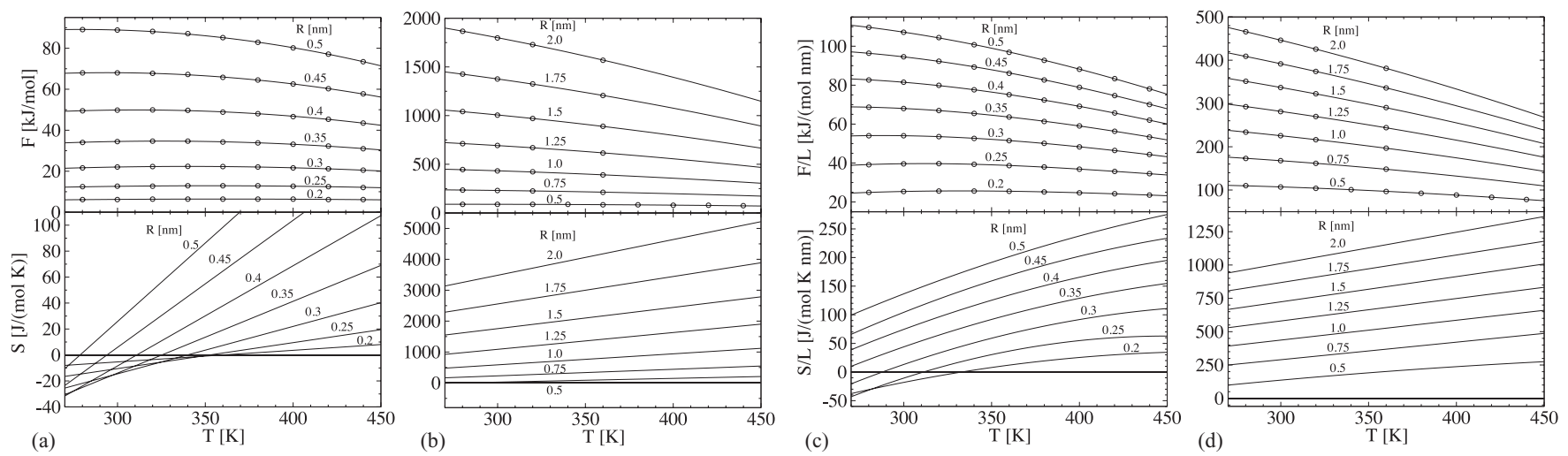

FIG. 14. Solvation free energies (top panels) and entropies (bottom panels) for solutes of different radii as a function of temperature. (a) and (b) Solvation free energies and entropies for spherical solutes. (c) and (d) Solvation free energies and entropies for cylindrical solutes. Open symbols show $F$ in the case of spherical solutes and $F / L$ in the case of cylindrical solutes. Lines are polynomial fits to the data. Entropies are obtained from the fits to the free energies by Eq. (19). All data are obtained by thermodynamic integration in SPC/E water at a constant pressure of $p=1$ bar.

zero crossings of the entropies) are only for the smallest radii within the studied temperature range. Consequently, most entropy intersections are not accessible. By defining the entropy convergence temperature $T_{S}^{*}$ for a given solute radius $R$ by $^{4}$

$$
S\left(R, T_{S}^{*}\right)=S\left(R+\delta R, T_{S}^{*}\right)
$$

with a radius increment of $\delta R=0.1 \mathrm{~nm}$, the different behavior of the spherical and cylindrical convergence temperatures is quantified in Fig. 15. Note, that we only show $T_{S}^{*}$ for the studied temperature range $T>280 \mathrm{~K}$. The maximum and subsequent decrease of the convergence temperatures for spheres with radii $R<0.1 \mathrm{~nm}$ is due to the finite $r \rightarrow 0$ limit of the Buckingham potential (see discussion above). The convergence temperature $T_{S}^{*}$ for spherical solutes is much higher than for cylindrical solutes of the same radius and for the cylindrical solutes the convergence temperature lies outside the considered temperature region for solutes with radii larger than $R \approx 0.18 \mathrm{~nm}$. This difference in convergence temperatures between spheres and cylinders reflects-among other factors- the different crossover radii $R^{*}$ for spheres and cylinders.

\section{CONCLUSION}

Extensive MD simulations for spherical and cylindrical solutes in SPC/E water yield a maximum of the solvation free energy per area, $F / A$, as a function of the solute radius $R$, which implies that the Tolman length characteriz-

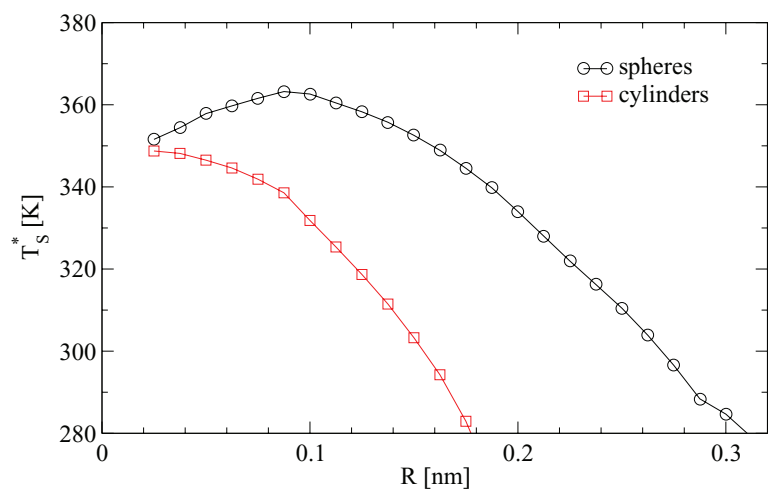

FIG. 15. Entropy convergence temperature $T_{S}^{*}$ defined by Eq. (29) for spherical (circles) and cylindrical (squares) solutes as a function of the solute radius $R$. The entropies are obtained from quadratic (spherical solutes) and cubic (cylindrical solutes) fits to the free energy data $F$ shown in Fig. 14. 
ing the water-hydrophobe interface is negative. This maximum occurs between radii of $R_{\max }=2 \mathrm{~nm}$ (for spheres at $T=300 \mathrm{~K}$ ) and $R_{\max }=0.8 \mathrm{~nm}$ (for cylinders at $T=360 \mathrm{~K}$ ). A simultaneous and consistent fit of sphere and cylinder solvation data yields a negative Tolman length of $\delta=-0.1 \mathrm{~nm}$ at $T=300 \mathrm{~K}$ for a hydrophobic surface, which means that droplets are favored over cavities, with important implications for current coarse-grained modelling. ${ }^{8}$ For cylinders in water at room temperature, the Tolman length $\delta \simeq-0.1 \mathrm{~nm}$, the radius $R_{\max } \simeq 1 \mathrm{~nm}$ where $F / A$ shows an extremum (which scales like the inverse spontaneous curvature, $R_{\max } \simeq c_{0}^{-1}$ ), and the crossover radius $R^{*} \simeq 0.5 \mathrm{~nm}$ between small-scale (where $F \sim V$ ) and large-scale solvation (where $F \sim A$ ) are quite similar, which requires a careful numerical analysis of the various crossover effects. In fact, the crossover radius $R^{*}$ is smaller for cylinders than for spheres and also decreases with increasing temperature, which makes cylinders particularly suited to study local curvature-based surface free-energy functionals. Our results suggest that for curvature radii $R>R_{\max }$, a curvature-based local interface free energy functional is valid for arbitrarily shaped solutes and the linear Tolman correction is dominant. For smaller radii, corrections have to be accounted for, either in the form of higher-order curvature terms (involving additional independent elastic constants) or in terms of empirical interpolation formulas that for a restricted class of geometries correctly account for the crossover to the small-scale solvation regime.

\section{ACKNOWLEDGMENTS}

Financial support of the Elitenetzwerk Bayern (CompInt) and computing time on the HLRB-II (Project pr28xe) of the Leibnitz-Rechenzentrum München are acknowledged.

${ }^{1}$ C. Tanford, The Hydrophobic Effect: Formation of Micelles and Biological Membranes, 1st ed. (Wiley-Interscience, New York, 1973).

${ }^{2}$ K. Lum, D. Chandler, and J. D. Weeks, J. Phys. Chem. B 103, 4570 (1999).

${ }^{3}$ D. Chandler, Nature 437, 640 (2005).

${ }^{4}$ H. S. Ashbaugh and L. R. Pratt, Rev. Mod. Phys. 78, 159 (2006).

${ }^{5}$ R. C. Tolman, J. Chem. Phys. 17, 333 (1949).

${ }^{6} \mathrm{~J}$. S. Rowlinson and B. Widom, Molecular Theory of Capillarity, 1st ed. (Clarendon, Oxford, 1982).

${ }^{7}$ W. Helfrich, Z. Naturforsch. C 28, 693 (1973).

${ }^{8}$ J. Dzubiella, J. Swanson, and J. McCammon, Phys. Rev. Lett. 96, 087802 (2006).
${ }^{9}$ H. S. Ashbaugh, J. Chem. Phys. 130, 204517 (2009).

${ }^{10}$ A. van Giessen and E. Blokhuis, J. Chem. Phys. 131, 164705 (2009).

${ }^{11}$ J. Henderson, Mol. Phys. 50, 741 (1983).

${ }^{12}$ A. Poniewierski and J. Stecki, J. Chem. Phys. 106, 3358 (1997).

${ }^{13}$ G. Graziano, Chem. Phys. Lett. 497, 33 (2010).

${ }^{14}$ M. Moody and P. Attard, J. Chem. Phys. 115, 8967 (2001).

${ }^{15}$ E. M. Blokhuis and J. Kuipers, J. Chem. Phys. 126, 054702 (2007).

${ }^{16}$ H. S. Ashbaugh and M. E. Paulaitis, J. Am. Chem. Soc. 123, 10721 (2001).

${ }^{17}$ F. Floris, J. Phys. Chem. B 109, 24061 (2005).

${ }^{18}$ D. M. Huang, P. L. Geissler, and D. Chandler, J. Phys. Chem. B 105, 6704 (2001).

${ }^{19}$ T. Yamamoto and S. Ohnishi, Phys. Chem. Chem. Phys. 13, 16142 (2011).

${ }^{20}$ E. M. Blokhuis, J. Groenewold, and D. H. Bedeaux, Mol. Phys. 96, 397 (1999).

${ }^{21}$ K. R. Mecke and S. Dietrich, Phys. Rev. E 59, 6766 (1999).

${ }^{22}$ E. Chacón and P. Tarazona, J. Phys. Condens. Matter 17, S3493 (2005).

${ }^{23}$ F. Sedlmeier, D. Horinek, and R. R. Netz, Phys. Rev. Lett. 103, 136102 (2009).

${ }^{24}$ R. L. Baldwin, Proc. Natl. Acad. Sci. U.S.A. 83, 8069 (1986).

${ }^{25}$ P. L. Privalov and S. J. Gill, Adv. Protein Chem. 39, 191 (1988).

${ }^{26}$ K. P. Murphy, P. L. Privalov, and S. J. Gill, Science 247, 559 (1990).

${ }^{27}$ S. Garde, G. Hummer, A. E. Garcia, M. E. Paulaitis, and L. R. Pratt, Phys. Rev. Lett. 77, 4966 (1996).

${ }^{28}$ G. Hummer, S. Garde, A. E. Garcia, M. E. Paulaitis, and L. R. Pratt, J. Phys. Chem. B 102, 10469 (1998).

${ }^{29}$ A. D. Robertson and K. P. Murphy, Chem. Rev. 97, 1251 (1997).

${ }^{30}$ F. Sedlmeier, D. Horinek, and R. R. Netz, J. Chem. Phys. 134, 055105 (2011).

${ }^{31}$ H. J. C. Berendsen, J. R. Grigera, and T. P. Straatsma, J. Phys. Chem. 91, 6269 (1987).

${ }^{32}$ J. R. Errington and A. Z. Panagiotopoulos, J. Phys. Chem. B 102, 7470 (1998).

${ }^{33}$ M. R. Shirts, J. W. Pitera, W. C. Swope, and V. S. Pande, J. Chem. Phys. 119, 5740 (2003).

${ }^{34}$ T. C. Beutler, A. E. Mark, R. C. Vanschaik, P. R. Gerber, and W. F. van Gunsteren, Chem. Phys. Lett. 222, 529 (1994).

${ }^{35}$ B. Widom, J. Chem. Phys. 39, 2808 (1963).

${ }^{36}$ P. Sindzingre, G. Ciccotti, C. Massobrio, and D. Frenkel, Chem. Phys. Lett. 136, 35 (1987).

${ }^{37}$ J. G. Kirkwood and F. P. Buff, J. Chem. Phys. 17, 338 (1949).

${ }^{38}$ B. Hess, C. Kutzner, D. van der Spoel, and E. Lindahl, J. Chem. Theory Comput. 4, 435 (2008).

${ }^{39}$ D. van der Spoel, E. Lindahl, B. Hess, G. Groenhof, A. E. Mark, and H. J. C. Berendsen, J. Comput. Chem. 26, 1701 (2005).

${ }^{40}$ H. J. C. Berendsen, J. Postma, W. F. van Gunsteren, A. Dinola, and J. Haak, J. Chem. Phys. 81, 3684 (1984).

${ }^{41}$ U. Essmann, L. Perera, M. L. Berkowitz, T. Darden, H. Lee, and L. Pedersen, J. Chem. Phys. 103, 8577 (1995).

${ }^{42}$ T. Darden, D. York, and L. Pedersen, J. Chem. Phys. 98, 10089 (1993).

${ }^{43}$ H. S. Ashbaugh, Chem. Phys. Lett. 477, 109 (2009).

${ }^{44}$ H. Reiss, H. Frisch, E. Helfand, and J. Lebowitz, J. Chem. Phys. 32, 119 (1960).

${ }^{45}$ D. Horinek, A. Herz, L. Vrbka, F. Sedlmeier, S. Mamatkulov, and R. Netz, Chem. Phys. Lett. 479, 173 (2009). 